Research Article

\title{
Simulation of the Dynamic Response to Sinusoidal Excitation of the Elastic Porous Metal Mesh Damper
}

\author{
Yichuan Shao $\mathbb{D}$, Shibin Song, Taia Xin, Jinyu Wang, and Xiaobo Xie \\ Engineering Research Center for Metal Rubber, School of Mechanical Engineering and Automation, Fuzhou University, \\ Fuzhou 350116, China \\ Correspondence should be addressed to Yichuan Shao; shaoyichuan612@fzu.edu.cn
}

Received 2 October 2020; Accepted 24 July 2021; Published 2 August 2021

Academic Editor: Adolfo Delgado

Copyright ( 2021 Yichuan Shao et al. This is an open access article distributed under the Creative Commons Attribution License, which permits unrestricted use, distribution, and reproduction in any medium, provided the original work is properly cited.

As a promising device of vibration isolation for rocket engine turbopumps, turbine engines, and other kinds of rotordynamic applications, the elastic porous metal mesh damper (MMD) has drawn large attention from researchers. It exhibits higher load capacity and environmental adaptivity than traditional viscoelastic damping materials owing to the excellent combination of metallic properties and the rubber-like damping performance. However, the design of a metal mesh damper relies heavily on the trial-and-error methodology for tuning fabrication parameters, which prevents it from widespread use in rotor-bearing applications. Therefore, efforts are directed toward forging an explicit link between fabrication parameters and the dynamic behaviors of MMDs in the present work. Quasistatic and dynamic mechanical tests are carried out to help determine the primary factor that influences the damping performance of the MMD and to demonstrate how the dynamic behaviors of MMDs evolve with the fabrication parameters. Furthermore, two different modeling methodologies, i.e., the FE method and the mixed damping approach, are used to predict the hysteresis behaviors of the dampers. The latter method not only properly reproduces the experimental results but also makes it possible to build an intuitive connection between the fabrication procedures and the dynamic mechanical behaviors of the MMDs. The orthogonal test results determine that the mesh density plays a dominant role in controlling both the load capacity and damping performance of the MMDs. By integrating mesh density and motion amplitude into the expression of parameters including stiffness coefficient, damping coefficient, and damping component factor, the mixed damping model demonstrates excellent predictive accuracy under different excitation conditions.

\section{Introduction}

The vibration damping of rocket engine turbopumps, gas turbine engines, and other electric components for aerospace shuttles has long been a major challenge for engineers due to the extreme environment those devices are exposed to. For instance, the devices could suffer from the pyrotechnic shock caused by separation events or experience low-frequency vibration amplification generated by the engine thrust and aerodynamic loads [1-3]. Furthermore, a working temperature tolerance of $-157^{\circ} \mathrm{C}+121^{\circ} \mathrm{C}$ is required for the vibration isolators to withstand the temperature change within the orbiting Space Shuttle.
Viscoelastic materials such as rubber and composites have been traditionally employed as vibration isolators for aerospace devices. However, deterioration and structure failure may frequently appear because of the poor working conditions, which considerably increase the maintenance cost of the vibration isolation system. Metal mesh dampers constructed by intertwined helix metal wires have been considered as alternatives to traditional viscoelastic dampers owing to the increasing need for a higher load capacity in extreme temperature conditions. By taking advantage of abundant dry friction joints within the complicated wire mesh structure, metal mesh dampers provide larger energy dissipation than rubber dampers [4]. Exhaustive experimental work has demonstrated the aerospace applications of 
metal mesh dampers fabricated with shape memory alloys (SMAs) [5-10].

A typical use of the radial direction of the metal mesh damper is the vibration control of the rotor-bearing system of turbomachinery [11], especially the turbopump, gas turbine engines, and turbochargers where high vibration amplitude could be produced over a wide range of frequency [12-15]. Reported results [16] demonstrate excellent vibration attenuation effects of metal mesh bearings on the motion amplitude of the rotor. San Andrés [17] implemented ring-shaped metal mesh foil bearings into an oil-free microturbomachinery and achieved a structural loss factor as high as 0.7. The bearing stiffness is found sensitive to the motion amplitude and frequency in this study. Lee's work [18] relates the damping capacity of the metal mesh damper to its density and suggests that higher density leads to higher stiffness and damping loss factor. Ryu [19] investigated the effects of preload on the damping characteristics of the metal mesh damper equipped on automotive turbochargers via static loading-unloading cycles. The test results indicate that preload has a significant impact on the loss factor of the metal mesh dampers with lower density.

Although promising results can be obtained by using metal mesh dampers, a large number of fabrication tests are usually required to optimize the performance of the final products. One can hardly predict the damping characteristics of the mesh damper based on experience because of the complicated interactions between individual wires [20]. Therefore, a predictive model based on either mathematics or microstructural considerations is necessary for widespread use in rotordynamic applications. Cao [21] simulated the static performance of metal mesh cushion by deconstructing it as close-packed piles of microcurved beams and further modeling the elastic behavior and Coulomb damping of the individual wires. However, the dynamic response of the metal mesh materials is quite distinct from the static behavior. Choudhry and Vance [22] introduced a dynamic model with consideration of factors including metal mesh ring geometry, displacement amplitude, axial thickness, and exciting frequency. Al-Khateeb [23] developed a model with stick-slip behaviors considered and predicted the variation of stiffness well. Recently, the hysteresis loop has frequently been used to characterize the dynamic behavior of metal mesh dampers. Hou [24] further developed this methodology by decomposing the hysteresis cycle into an asymmetric nonlinear elastic restoring force, a viscous damping force, and a bilinear hysteresis Coulomb friction force. By doing so, hysteresis curves tested with different amplitudes and exciting frequencies at temperatures between $20^{\circ} \mathrm{C}$ and $300^{\circ} \mathrm{C}$ can be well predicted by the model.

As revealed by the literature [25], the hysteresis behavior is not only affected by the ambient temperatures, amplitude, and exciting frequency but also dependent upon the fabrication parameters of the damper, such as density, crisscross angle, and wire diameter. Those factors are usually intertwined and interaffected, which makes it hard to establish an explicit relationship between those parameters and the hysteresis behaviors. Therefore, of particular interest to the authors is to determine the primary factor that affects the hysteresis behavior. An additional concern is modeling this hysteresis behavior with the primary factor involved so that the simulation results can automatically respond to the change of processing parameters or exciting conditions.

\section{Experimental Setup and Test Methods}

2.1. Fabrication of MMD. MMDs are typically constructed either by spiral metal thread or by knitted metal mesh. The former type of MMDs usually presents chaotic wire patterned structures, while the latter type has a layer-structured typology [26]. Because the spiral thread-based type is chosen as the objective of the present study, all the tested results and conclusions from this paper might not apply to the knitted metal mesh dampers.

As illustrated in Figure 1, the following procedures were carried out to fabricate MMDs:

(1) 304 stainless steel wire was chosen as the raw material to fabricate all the dampers used in the present work.

(2) The steel wire was encircled into a tight helical wire which is prepared for the subsequent fabrication steps.

(3) After that, the helix wire was pretensioned to some extent to make sure the pitch is equal from one loop to another and then wrapped around a mandrel and arranged in a crisscross pattern to obtain a rough porous soft roll. The angle between the helix thread and the transverse direction of the mandrel is taken as $\theta$ for convenience.

(4) Finally, the soft roll was put into a mold and highly compressed until the final damper geometry was reached.

(5) The outer diameter, inner diameter, and height of the dampers used in the present work are $22 \mathrm{~mm}$, $12 \mathrm{~mm}$, and $15 \mathrm{~mm}$, respectively.

Experience shows that fabrication parameters including the wire diameter, the crossing angle of helix wire, and the density are all capable of adjusting the stiffness and loss factor of the MMD. As shown in Table 1, fabrication parameters representative of typical situations of manufacturing ringshaped MMDs are tested in the present work.

\subsection{Quasistatic Compression Tests}

2.2.1. Test Method. Static compression tests of the MMDs were conducted by using a WDW-T200 electromechanical universal testing machine with a constant loading speed of $5 \mathrm{~mm} / \mathrm{min}$ and a maximum load of $2 \mathrm{kN}$. As shown in Figure 2(a), only the radial direction of the sample was tested in the present work. The outer surface of the damper is subjected to the load from the fixture whereas the inner surface is constrained by the mandrel.

An orthogonal test design was used to help determine the key factor that mainly influences the equivalent stiffness and loss factor of the MMDs. In this test, attention was focused on four factors including mesh density $\rho$, crisscross 
(2) Tight helix wire
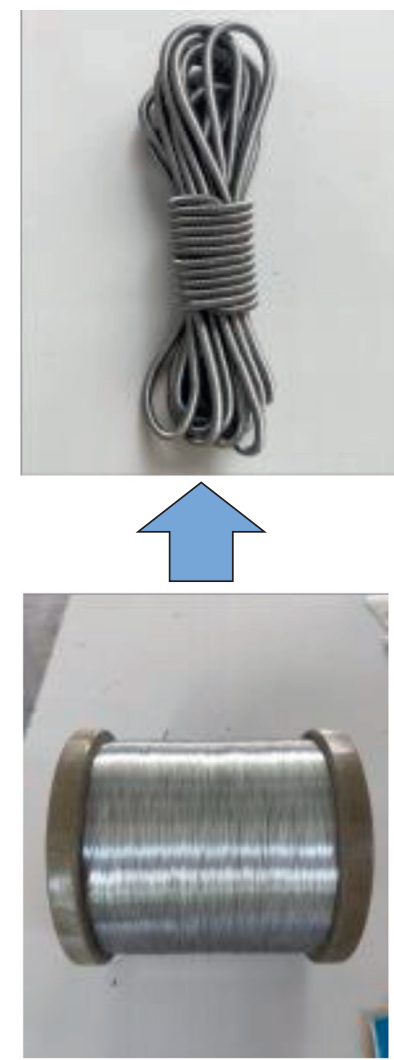

(1) 304 stainless steel wire
(3) Rough porous soft roll
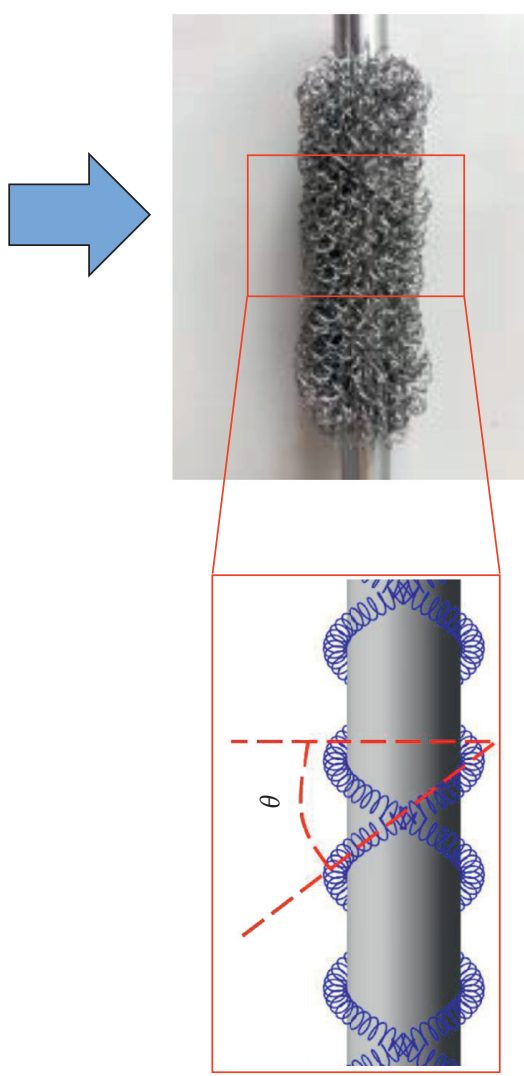

Crisscross angle

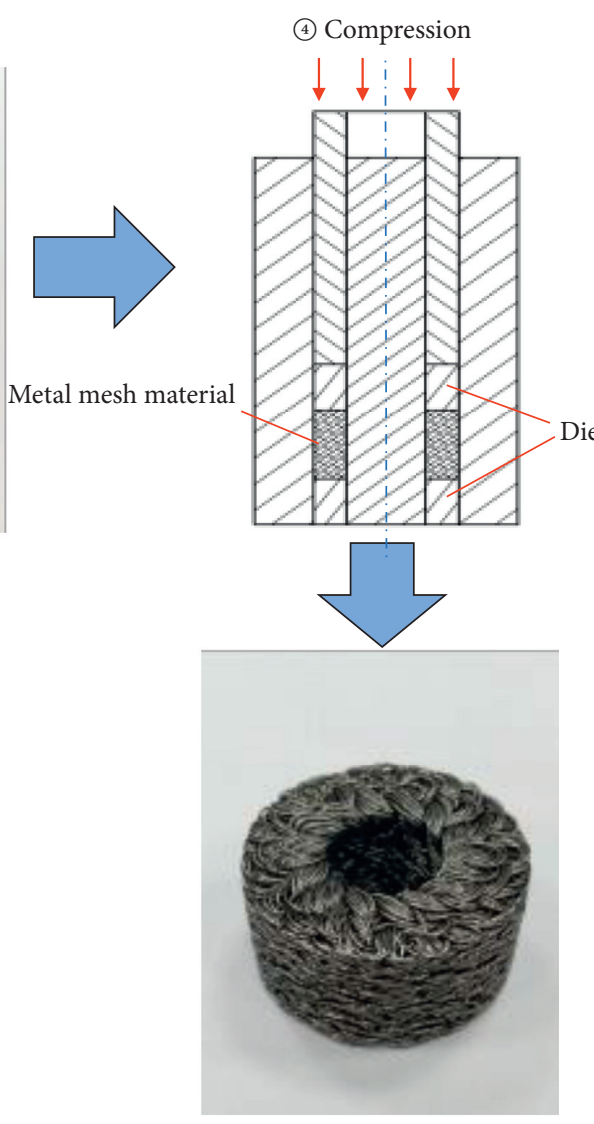

(5) Metal mesh damper

Figure 1: Fabrication of the metal mesh damper.

TABLE 1: The tested fabrication parameters.

\begin{tabular}{lc}
\hline Parameter & Value \\
\hline Wire diameter $(\mathrm{mm})$ & $0.1,0.15,0.3$ \\
Crisscross angle $\left({ }^{\circ}\right)$ & $15,30,45,60,75$ \\
Mesh density $\left(\mathrm{g} / \mathrm{cm}^{3}\right)$ & $2.0,2.5,3.0,3.5,4.0$ \\
\hline
\end{tabular}

angle $\theta$, wire diameter $d$, and test load $F$. The maximum number of levels considered in the test is limited by the localmade helix thread machine tool which is only applicable for wires with diameters $0.1 \mathrm{~mm}, 0.15 \mathrm{~mm}$, and $0.3 \mathrm{~mm}$. As a result, an $L_{9}\left(3^{4}\right)$ orthogonal array was adopted so that each of the factors has 3 levels, as shown in Table 2 .

2.2.2. Characterization of Static Stiffness and Loss Factor. As shown in Figure 3(a), metal mesh materials typically demonstrate nonlinear loading and unloading curves, of which the tangent stiffness is not a comprehensive description of the hysteresis behavior. Instead, equivalent stiffness $k_{e q}$ is used here to characterize the load capacity of the MMDs, as depicted in Figure 3(b). The area under the middle line of the hysteresis loop, which is also called elastic potential $U$, equals the area under the line with a slope of $k_{e q}$ :

$$
k_{\mathrm{eq}}=\frac{2 U}{x_{\max }^{2}},
$$

where $x_{\max }$ is the maximum loading displacement.

The loss factor $\eta_{s}$ can be calculated by

$$
\eta_{s}=\frac{\Delta W}{\pi U}
$$

where the energy dissipation $\Delta W$ equals the area of the hysteresis loop.

\subsection{Dynamic Mechanical Test}

2.3.1. Test Method. The dynamic test of the MMDs was carried out using an SDS-200 hydraulic universal testing machine which provides a maximum load of $200 \mathrm{kN}$ and a maximum displacement of $\pm 50 \mathrm{~mm}$. As shown in Figure 2(b), the ring-shaped damper was positioned in the fixtures to simulate the practical vibration conditions and then gripped in the testing machine. The sine test protocol was performed with a peak amplitude of $0.1 \mathrm{~mm}$ and a constant frequency of $1 \mathrm{~Hz}$. It is undeniable that the stiffness of MMD is frequency-dependent [17]. However, the change of hysteresis loop versus frequency is hard to be noticed when the frequency range narrows down to $1 \sim 10 \mathrm{~Hz}$ [27]. In the present work, our efforts are directed toward modeling the effects of fabrication parameters, and hence, a fixed frequency was adopted in the test. 


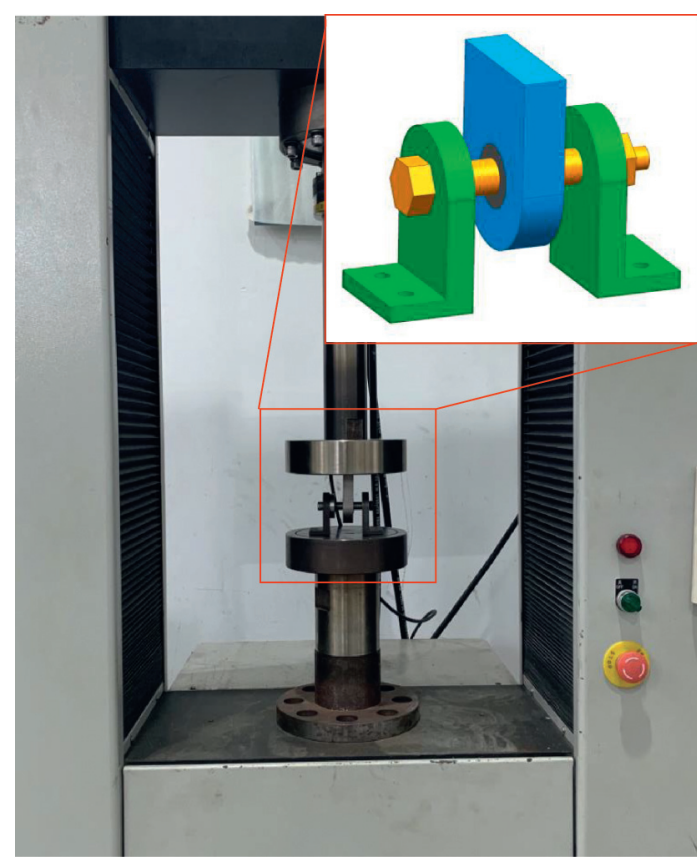

(a)

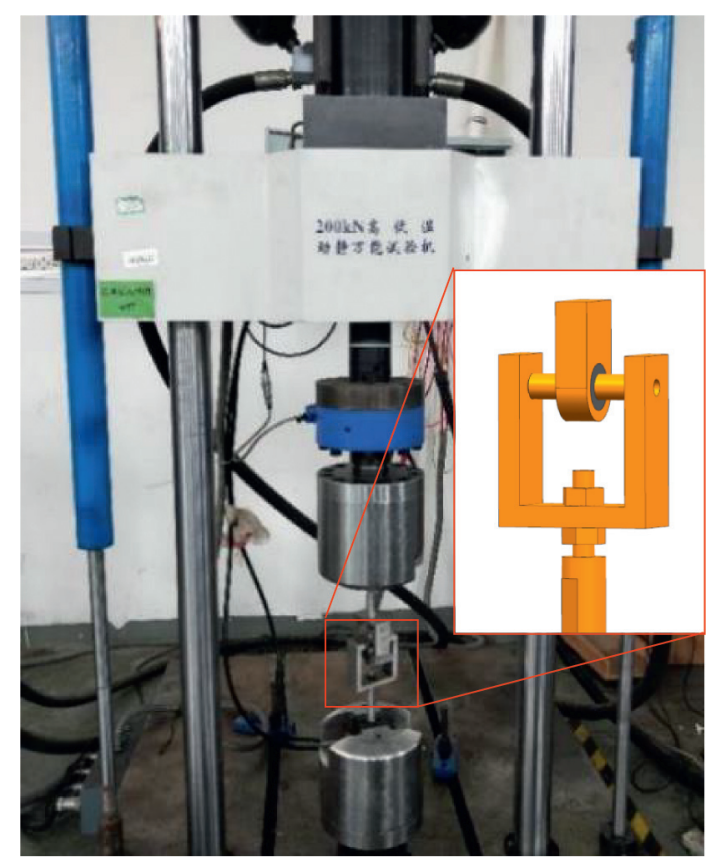

(b)

FIgure 2: Mechanical test setup of the MMDs. (a) Quasistatic compression. (b) Dynamic mechanical test.

TABLE 2: Orthogonal array for static compression tests of MMDs.

\begin{tabular}{lcccc}
\hline Factor & & \multicolumn{2}{c}{ Level } & \\
& & 1 & 2 & 3.0 \\
\hline Mesh density, $\rho\left(\mathrm{g} / \mathrm{cm}^{3}\right)$ & $A$ & 2.0 & 4.0 & 4.0 \\
Crisscross angle, $\theta\left({ }^{\circ}\right)$ & $B$ & 15 & 0.15 & 75 \\
Wire diameter, $d(\mathrm{~mm})$ & $C$ & 0.1 & 1.5 & 0.3 \\
Test load, $F(\mathrm{kN})$ & $D$ & 1 & 2 \\
\hline
\end{tabular}

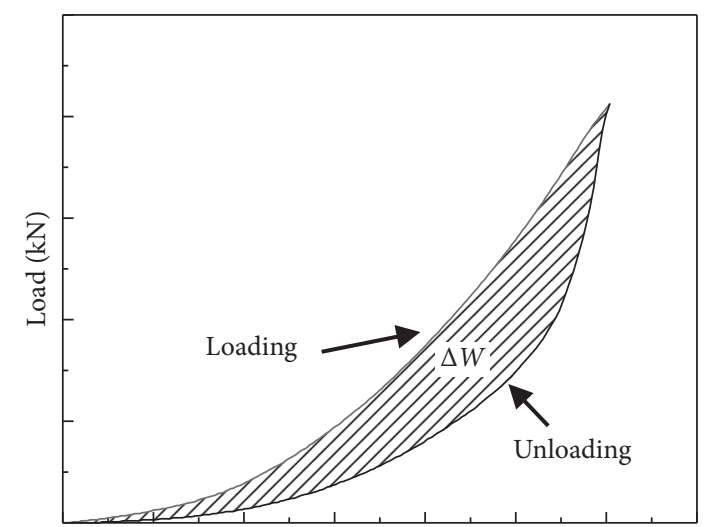

Displacement (mm)

(a)

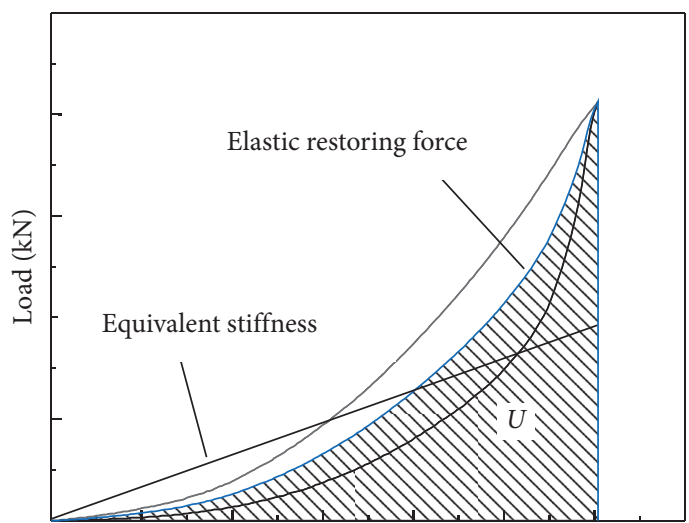

Displacement (mm)

(b)

FIgURe 3: Characterization of static stiffness and loss factor. (a) Hysteresis behavior of MMD under static load. (b) Elastic restoring force and equivalent stiffness of an MMD. 
2.3.2. Characterization of Dynamic Stiffness and Loss Factor. Dynamic stiffness can be viewed as the ability of the MMD to resist an external oscillatory load. It differs from static stiffness concerning the calculations and can be expressed by the load that is required to produce unit displacement on the damper, i.e.,

$$
K=\frac{F_{\max }-F_{\min }}{2 X_{0}}
$$

where the $F_{\max }$ and $F_{\min }$ are the maximum and minimum restoring force sampled from the hysteresis curve and $X_{0}$ is the peak motion amplitude of the test.

The loss factor can be written in the form of energy dissipation, $\Delta W$, divided by the elastic potential, $W$, within a complete cycle of vibration, which gives

$$
\eta_{d}=\frac{\Delta W}{2 \pi W}
$$

where the energy dissipation can be obtained by

$$
\begin{aligned}
\Delta W & =\int F d x \\
& =-\omega X_{0} \int_{0}^{T} F \sin (\omega t+\alpha) \mathrm{d} t \\
& =-\frac{2 \pi X_{0}}{N} \sum_{i=1}^{N} F_{i} \sin \left(\frac{2 \pi i}{N}+\alpha\right),
\end{aligned}
$$

and the elastic potential is given by

$$
W=\frac{1}{2} K X_{0}^{2}
$$

Substituting equations (5) and (6) into (4), the dynamic loss factor can be written as

$$
\eta_{d}=-\frac{4 f}{f_{0}\left(F_{\max }-F_{\min }\right)} \sum_{i=1}^{N} F_{i} \sin \left(\frac{2 \pi i}{N}+\alpha\right) .
$$

\section{Modeling Procedures}

\subsection{Finite Element Modeling}

3.1.1. Material Models. It is necessary to use a hyperelastic model in conjunction with a viscoelastic model for describing the hysteresis behavior of metal mesh material [28]. The Mooney-Rivlin model [29] was adopted to simulate the hyperelasticity of the MMD. The mechanical strain energy can be expressed as a sum of invariants as follows:

$$
W=\Sigma_{i} \Sigma_{j} C_{i j}\left(I_{1}-3\right)^{i}\left(I_{2}-3\right)^{j}+D(J-1)^{2},
$$

where $C_{i j}$ and $D$ are empirically determined material constants, $I 1$ and $I 2$ are the first and the second invariants of the deviatoric component of the left Cauchy-Green deformation tensor, and $J$ is the determinant of the deformation gradient $F$. The number of terms in the expansion depends on the application's accuracy requirements. For instance, the first few terms of the series can be written as

$$
W=C_{10}\left(I_{1}-3\right)+C_{01}\left(I_{2}-3\right)+C_{11}\left(I_{1}-3\right)\left(I_{2}-3\right)+C_{20}\left(I_{1}-3\right)^{2}+\cdots+D(J-1)^{2}
$$
by

The Cauchy stress for the Mooney-Rivlin model is given

$$
\boldsymbol{\sigma}=\frac{2}{J}\left(C_{10}+C_{01} I_{1}\right) \mathbf{b}^{*}-\frac{2 C_{01}}{J}\left(\mathbf{b}^{*}\right)^{2}+\left[2 D(J-1)-\frac{2 I_{1} C_{10}}{3 J}-\frac{4 I_{2} C_{01}}{3 J}\right] \mathbf{I},
$$

where $\mathbf{b}^{*}=(J)^{-2 / 3} \mathbf{b}$ and $\mathbf{b}$ is a dimensionless symmetric fourth-order tensor of material parameters.

The integral formulation for linear isotropic viscoelasticity is given by

$$
\boldsymbol{\sigma}(t)=\int_{0}^{t} 2 G(t-\tau) \frac{\mathrm{d} \mathbf{e}}{\mathrm{d} \tau} \mathrm{d} t+\mathbf{I} \int_{0}^{t} K(t-\tau) \frac{\mathrm{d} \phi}{\mathrm{d} \tau} \mathrm{d} t,
$$

where $\mathbf{e}$ and $\phi$ are the mechanical deviatoric and volumetric strains and $K$ and $G$ are the bulk modulus and the shear modulus, which are functions of the reduced time $t$. The relaxation functions $K(t)$ and $G(t)$ can be defined individually in terms of a series of exponentials known as the Prony series:

$$
\begin{aligned}
& K(t)=K_{\infty}+\sum_{i=1}^{n_{K}} K_{i} e^{-t / \tau_{i}^{K}}, \\
& G(t)=G_{\infty}+\sum_{i=1}^{n_{G}} G_{i} e^{-t / \tau_{i}^{G}},
\end{aligned}
$$

where $K \infty$ and $G \infty$ represent the long-term bulk and shear moduli; $n_{K}$ and $n_{G}$ are the number of terms of the bulk and shear moduli; and $\tau_{i}^{K}$ and $\tau_{i}^{G}$ are relaxation times of the bulk and shear moduli.

3.1.2. Mesh and Boundary Conditions. All the FE simulations were carried out using ANSYS workbench software. The static structural and transient structural modules were 
used for the quasistatic compression and dynamic loading simulations, respectively. The geometry of the FE analysis was modeled based on the MMDs and fixtures used in the experiment and then discretized by hexahedral elements, as shown in Figure 4.

The boundary conditions of the quasistatic compression model and the dynamic model are the same as the experimental conditions. It is worth mentioning that the surfaces of the metal mesh ring and the mandrel are allowed to be separated, and the frictionless condition is defined for this contact pair.

To simulate the dynamic loading conditions, a sinusoidal excitation is applied as displacement as $A \sin (\pi, t)$ to the upper plane of the damper fixture, as shown in Figure 4. Both ends of the mandrel are constrained by the fixture to keep the loading condition identical to the experiment. The inner surface of the damper is not bonded together with the outer surface of the mandrel and a frictionless definition is applied.

3.2. Nonlinear Elastic Mixed Damping Model. The hysteresis behavior of metal mesh damper can be decomposed into two different terms including asymmetric nonlinear elastic restoring force, $F_{k}(y)$, and nonlinear damping force, $F_{c}(y, \dot{y})$ [30], which gives

$$
\begin{aligned}
F(y(t), \dot{y}(t)) & =F_{k}(y)+F_{c}(y, \dot{y}) \\
& =k_{1} y(t)+k_{3} y^{3}(t)+c|\dot{y}(t)|^{\alpha} \operatorname{sgn}(\dot{y}(t)),
\end{aligned}
$$

where $k_{1}$ is combined with $k_{3}$ to describe the nonlinear elastic restoring behavior, and $c$ and $\alpha$ are used to define the type of damping force involved. Apparently, a greater value of $\alpha$ would lead to a higher sensitivity of damping force to the velocity. Furthermore, equation (13) can be expressed as Coulomb friction force if $\alpha$ equals 0 and can be simplified as a definition of linear damping force if $\alpha$ equals 1 . Mixing of different damping force components would appear if $\alpha$ is greater than 0 yet smaller than 1 . The identification of parameters $k_{1}, k_{3}, c$, and $\alpha$ is required to describe the hysteresis behavior of MMDs.

By fitting the hysteresis loops of MMDs fabricated with various densities against a 3-order polynomial,

$$
F_{\mathrm{k}}(y)=a_{0}+a_{1} y+a_{2} y^{2}+a_{3} y^{3}
$$

we can obtain the first-order stiffness coefficient $k_{1}=a_{1}$ and the third-order stiffness coefficient $k_{3}=a_{3}$, respectively. $k_{1}$ and $k_{3}$ can be further written as functions of excitation conditions and fabrication parameters, i.e., $k_{1}=k_{1}(X, Y)$, and $k_{3}=k_{3}(X, Y)$.

Once the term $F_{k}(y)$ is determined, the nonlinear damping force can be calculated by subtracting $F_{k}(y)$ from the hysteresis response $f_{k}$, i.e.,

$$
F_{c}\left(y_{k}, \dot{y}_{k}\right)=f_{k}-k_{1} y_{k}-k_{3} y_{k}^{3} .
$$

Consequently, coefficients $c$ and $\alpha$ can be identified by using a Gauss-Newton approach.

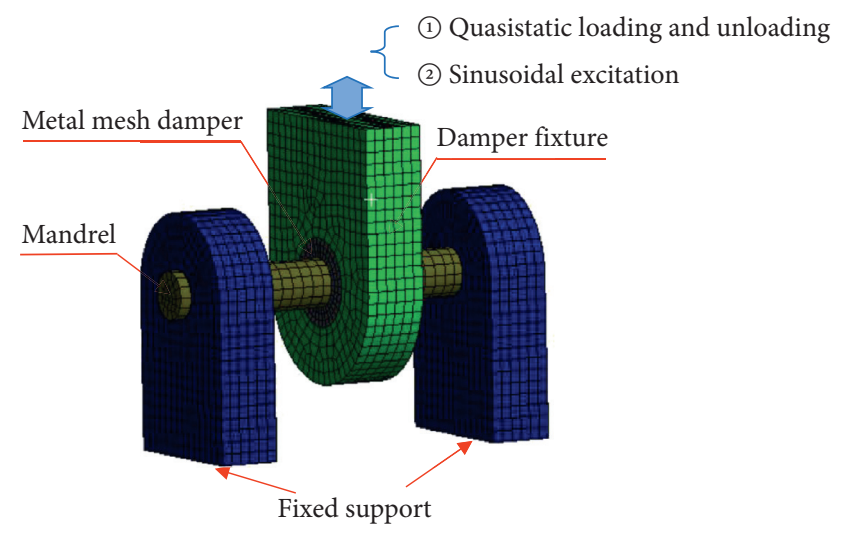

FIGURE 4: FE models for the static compression and dynamic loading of the MMD.

\section{Results}

4.1. Orthogonal Test Results. The key factor that primarily influences the loss factor and stiffness of the MMDs is determined by ordering the importance of all the four factors involved via the orthogonal test. As shown in Table 3, the statistical test parameter of factor set $x$ can be calculated by determining the sum of all the test result indices $K_{x y}$ containing level $y$ of factor $x$, and thus, a range analysis can be conducted by calculating the difference between the maximum and minimum values of $K_{x y}$.

By comparing and ordering the $R$ values for indices $\eta_{0}$ and $K_{e q}$, it is intuitive to determine the primary factor that affects the performance of the MMDs. As for the loss factor, the order of significance is test load $>$ mesh density $>$ crisscross angle $>$ wire diameter. For the equivalent stiffness, the order of significance is mesh density $>$ test load $>$ crisscross angle $>$ wire diameter.

4.2. Parameter Identification of the FE Model. The FE model introduced in Section 3.1 is fitted against the compression curve tested with a displacement of $0.5 \mathrm{~mm}$. A set of Mooney-Rivlin parameters for different mesh densities are obtained as listed in Table 4. The Prony shear relaxation parameters are obtained as shown in Table 5.

Prony shear relaxation parameters as shown in Table 5 are calibrated based on published results to ensure that the curves are well fitted.

\subsection{Parameter Identification of the Mixed Damping Model.} By fitting hysteresis loops of MMDs against equation (14), we can obtain the first-order and the third-order nonlinear stiffness coefficients as the following matrix.

The matrix of $k_{1}$ and $k_{3}$ values presented in Tables 6 and 7 can be subsequently plotted as functions of mesh density and amplitude, as shown in Figures 5(a) and 5(b), respectively.

The discrete points shown in Figure 5 are then fitted against a continuous binary function $k(\rho, A)$ to describe the evolution of $k$ values with mesh density and amplitude, which gives 
TABLE 3: Orthogonal test results of quasistatic compression of the MMDs.

\begin{tabular}{|c|c|c|c|c|c|c|}
\hline \multirow[b]{2}{*}{ No. } & \multicolumn{4}{|c|}{ Factor and level } & \multicolumn{2}{|c|}{ Index } \\
\hline & $\begin{array}{c}\text { Density } \\
\text { A }\end{array}$ & $\begin{array}{c}\text { Crisscross angle } \\
\text { B }\end{array}$ & $\begin{array}{c}\text { Wire diameter } \\
\text { C }\end{array}$ & $\begin{array}{c}\text { Test load } \\
\text { D }\end{array}$ & $\begin{array}{c}\text { Loss factor } \\
\eta_{0}\end{array}$ & $\begin{array}{c}\text { Equivalent stiffness } \\
K_{e q}\end{array}$ \\
\hline 1 & 1 & 1 & 1 & 1 & 0.0281 & 0.022 \\
\hline 2 & 1 & 2 & 2 & 2 & 0.09 & 0.0566 \\
\hline 3 & 1 & 3 & 3 & 3 & 0.206 & 0.07 \\
\hline 4 & 2 & 1 & 2 & 3 & 0.0681 & 0.129 \\
\hline 5 & 2 & 2 & 3 & 1 & 0.0462 & 0.215 \\
\hline 6 & 2 & 3 & 1 & 2 & 0.0113 & 0.1433 \\
\hline 7 & 3 & 1 & 3 & 2 & 0.0279 & 0.3462 \\
\hline 8 & 3 & 2 & 1 & 3 & 0.0582 & 0.1779 \\
\hline 9 & 3 & 3 & 2 & 1 & 0.043 & 0.48 \\
\hline \multicolumn{7}{|c|}{ Range analysis for the index $\eta_{0}(x=A, B, C, D)$} \\
\hline$\Sigma K_{x 1}$ & 0.3241 & 0.1241 & 0.09 & 0.1173 & & \\
\hline$\Sigma K_{x 2}$ & 0.1256 & 0.1944 & 0.2011 & 0.1292 & & \\
\hline$\sum K_{x 3}$ & 0.1291 & 0.259 & 0.28 & 0.3323 & & \\
\hline$R$ & 0.1985 & 0.1349 & 0.19 & 0.215 & & \\
\hline \multicolumn{7}{|c|}{ Range analysis for the index $K_{e q}(x=A, B, C, D)$} \\
\hline$\sum K_{x 1}$ & 0.1486 & 0.4972 & 0.3432 & 0.717 & & \\
\hline$\sum K_{x 2}$ & 0.4873 & 0.4495 & 0.6656 & 0.5461 & & \\
\hline$\sum K_{x 3}$ & 1.0041 & 0.6933 & 0.6312 & 0.3769 & & \\
\hline$R^{n j}$ & 0.8555 & 0.2438 & 0.3224 & 0.3401 & & \\
\hline
\end{tabular}

TABle 4: Mooney-Rivlin parameters adopted for the FE model.

\begin{tabular}{|c|c|c|c|c|}
\hline \multirow{2}{*}{ Density $\left(\mathrm{g} / \mathrm{cm}^{3}\right)$} & \multicolumn{4}{|c|}{ Material parameter $(\mathrm{MPa})$} \\
\hline & C10 & C01 & $C 11$ & $D 1$ \\
\hline 2.0 & 1.9 & -0.5 & 31 & 0.35 \\
\hline 2.5 & 60 & -54 & 100 & 0.35 \\
\hline 3.0 & 4 & -0.1 & 60 & 0.35 \\
\hline 3.5 & 60 & -54 & 90 & 0.35 \\
\hline 4.0 & 40 & -14 & 36 & 0.35 \\
\hline
\end{tabular}

TABLE 5: Prony shear relaxation parameters adopted for the FE model.

\begin{tabular}{lcccc}
\hline Parameter & $a_{1}$ & $t_{1}$ & $a_{2}$ & $t_{2}$ \\
\hline Value & 0.5548 & 0.4 & 0.4336 & 0.2 \\
\hline
\end{tabular}

TABLE 6: $k_{1}$ values identified for hysteresis loops of MMDs fabricated with various densities.

\begin{tabular}{|c|c|c|c|c|c|}
\hline \multirow{2}{*}{ Amplitude } & \multicolumn{5}{|c|}{$k_{1}$ values of MMDs with different densities $(\mathrm{kN} / \mathrm{mm})$} \\
\hline & $2.0 \mathrm{~g} / \mathrm{cm}^{3}$ & $2.5 \mathrm{~g} / \mathrm{cm}^{3}$ & $3.0 \mathrm{~g} / \mathrm{cm}^{3}$ & $3.5 \mathrm{~g} / \mathrm{cm}^{3}$ & $4.0 \mathrm{~g} / \mathrm{cm}^{3}$ \\
\hline $0.05 \mathrm{~mm}$ & 0.3365 & 0.2235 & 1.5273 & 4.3051 & 7.5503 \\
\hline $0.10 \mathrm{~mm}$ & 0.2823 & 0.3538 & 1.0539 & 2.5246 & 8.3948 \\
\hline $0.15 \mathrm{~mm}$ & 0.2957 & 0.2490 & 0.7007 & 2.1466 & 8.8699 \\
\hline $0.20 \mathrm{~mm}$ & 0.4368 & 0.5272 & 0.5333 & 1.7452 & 8.9133 \\
\hline $0.25 \mathrm{~mm}$ & 0.6376 & 0.9585 & 0.4655 & 1.2470 & 9.3548 \\
\hline
\end{tabular}


TABLE 7: $k_{3}$ values identified for hysteresis loops of MMDs fabricated with various densities.

\begin{tabular}{|c|c|c|c|c|c|}
\hline \multirow{2}{*}{ Amplitude } & \multicolumn{5}{|c|}{$k_{3}$ values of MMDs with different densities $(\mathrm{kN})$} \\
\hline & $2.0 \mathrm{~g} / \mathrm{cm}^{3}$ & $2.5 \mathrm{~g} / \mathrm{cm}^{3}$ & $3.0 \mathrm{~g} / \mathrm{cm}^{3}$ & $3.5 \mathrm{~g} / \mathrm{cm}^{3}$ & $4.0 \mathrm{~g} / \mathrm{cm}^{3}$ \\
\hline $0.05 \mathrm{~mm}$ & 86.7 & 151.1 & 131.9 & 185.9 & 160.9 \\
\hline $0.10 \mathrm{~mm}$ & 24.2 & 105.4 & 10.5 & 125.6 & 180.5 \\
\hline $0.15 \mathrm{~mm}$ & 11.9 & 54 & 10.1 & 46.5 & 5.2 \\
\hline $0.20 \mathrm{~mm}$ & 11.2 & 39.6 & 7.4 & 21.5 & 15.7 \\
\hline $0.25 \mathrm{~mm}$ & 9.4 & 34.9 & 4.7 & 8.1 & 18.5 \\
\hline
\end{tabular}

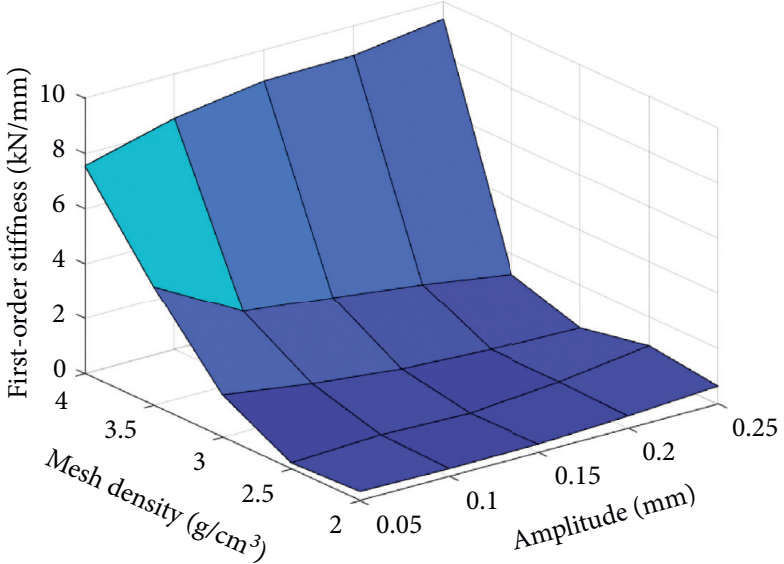

(a)

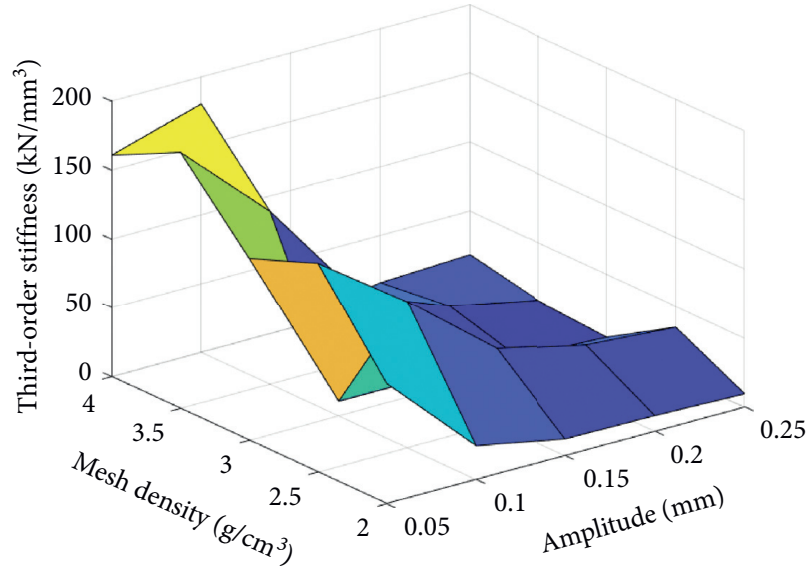

(b)

FIGURE 5: Stiffness coefficient plotted as a function of mesh density and amplitude. (a) $k_{1}$ and (b) $k_{3}$.

It is noteworthy that the maximum order of $A$ in equation (16) is limited to a low level. In so doing, the dependency of $k_{1}$ on amplitude can be considerably reduced to ensure that the discrete points are well fitted.

The nonlinear damping force can be obtained via equation (15), and then, we obtain the matrix of damping coefficient $c$ as shown in Table 8 .

The matrix of damping coefficient $c$ presented in Table 8 can be plotted as a function of mesh density and amplitude, as illustrated in Figure 6.

The discrete points shown in Figure 6 are then fitted against a continuous binary function $c(\rho, A)$ to describe the evolution of $c$ values with mesh density and amplitude, which gives

$$
\begin{aligned}
c(\rho, A)= & -6.705+9.596 A+6.924 \rho+0.7754 A^{2}-7.209 A \cdot \rho \\
& -2.286 \rho^{2}+0.1469 A^{3}+1.297 A^{2} \cdot \rho+0.2459 A \cdot \rho^{2} .
\end{aligned}
$$

The identification of the damping component factor, $\alpha$, is similar to that of the damping coefficient $c$. First, a matrix of $\alpha$ can be obtained via equation (15), as shown in Table 9.

The matrix of $\alpha$ values presented above can be plotted as a function of mesh density and amplitude, as illustrated in Figure 7.

The discrete points shown in Figure 7 are then fitted against a continuous binary function $\alpha(\rho, A)$ to describe the evolution of $\alpha$ values with mesh density and amplitude, which gives
TABLE 8: $c$ values identified for hysteresis loops of MMDs fabricated with various densities.

\begin{tabular}{lccccc}
\hline \multirow{2}{*}{ Amplitude } & \multicolumn{5}{c}{$c$ values of MMDs with different densities $(\mathrm{kN} \cdot \mathrm{s} / \mathrm{mm})$} \\
& $2.0 \mathrm{~g} / \mathrm{cm}^{3}$ & $2.5 \mathrm{~g} / \mathrm{cm}^{3}$ & $3.0 \mathrm{~g} / \mathrm{cm}^{3}$ & $3.5 \mathrm{~g} / \mathrm{cm}^{3}$ & $4.0 \mathrm{~g} / \mathrm{cm}^{3}$ \\
\hline $0.05 \mathrm{~mm}$ & 0.0235 & 0.0694 & 0.1397 & 0.1436 & 0.1107 \\
$0.10 \mathrm{~mm}$ & 0.0409 & 0.0899 & 0.1114 & 0.1359 & 0.2937 \\
$0.15 \mathrm{~mm}$ & 0.0446 & 0.1339 & 0.1252 & 0.1452 & 0.4147 \\
$0.20 \mathrm{~mm}$ & 0.0657 & 0.1657 & 0.1219 & 0.1320 & 0.5329 \\
$0.25 \mathrm{~mm}$ & 0.0899 & 0.2247 & 0.1263 & 0.1280 & 0.6592 \\
\hline
\end{tabular}

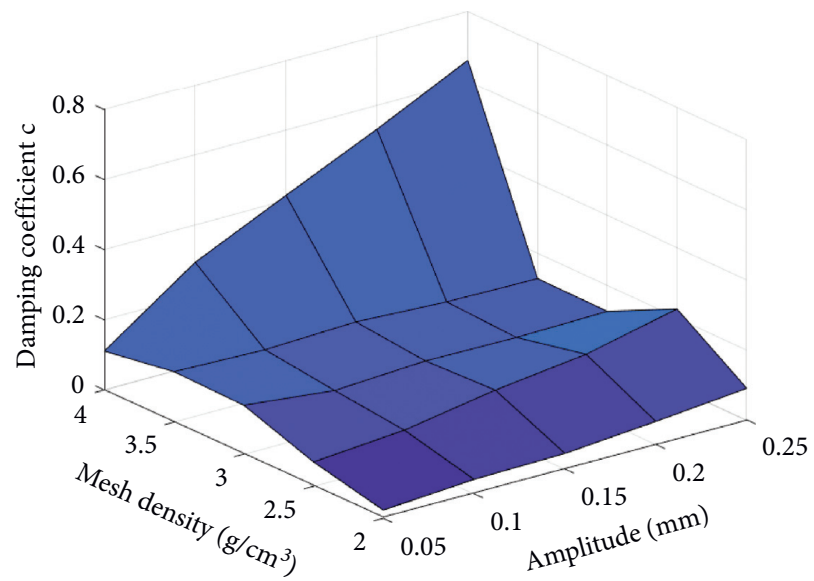

FIGURE 6: $c$ values plotted as a function of mesh density and amplitude. 
TABLE 9: $\alpha$ Values identified for hysteresis loops of MMDs fabricated with various densities.

\begin{tabular}{|c|c|c|c|c|c|}
\hline \multirow{2}{*}{ Amplitude } & \multicolumn{5}{|c|}{$\alpha$ Values of MMDs with different densities } \\
\hline & $2.0 \mathrm{~g} / \mathrm{cm} 3$ & $2.5 \mathrm{~g} / \mathrm{cm} 3$ & $3.0 \mathrm{~g} / \mathrm{cm} 3$ & $3.5 \mathrm{~g} / \mathrm{cm} 3$ & $4.0 \mathrm{~g} / \mathrm{cm} 3$ \\
\hline $0.05 \mathrm{~mm}$ & 0.4014 & 0.5571 & 0.4429 & 0.4187 & 0.2569 \\
\hline $0.10 \mathrm{~mm}$ & 0.4441 & 0.4797 & 0.3969 & 0.3370 & 0.3279 \\
\hline $0.15 \mathrm{~mm}$ & 0.3930 & 0.4461 & 0.3820 & 0.3516 & 0.2053 \\
\hline $0.20 \mathrm{~mm}$ & 0.3736 & 0.4304 & 0.3869 & 0.3143 & 0.1806 \\
\hline $0.25 \mathrm{~mm}$ & 0.3724 & 0.2722 & 0.3941 & 0.2484 & 0.1581 \\
\hline
\end{tabular}

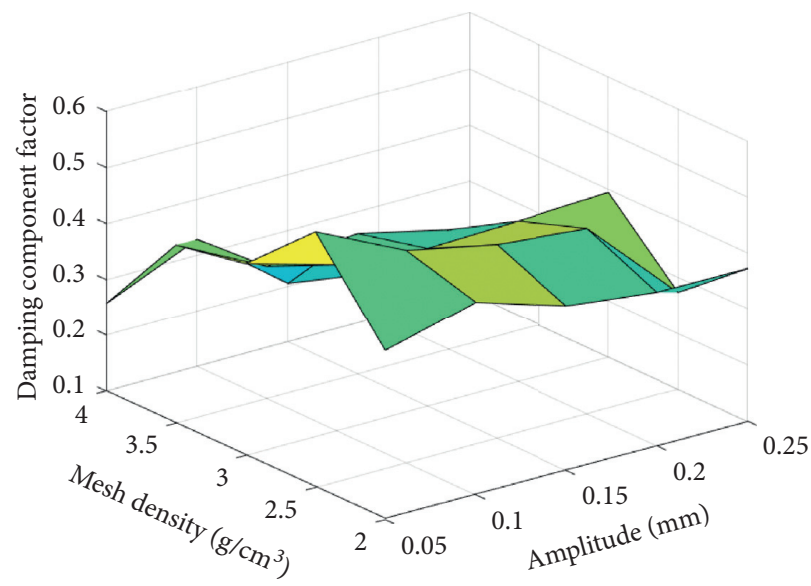

Figure 7: $\alpha$ Values plotted as a function of mesh density and amplitude.

$$
\begin{aligned}
\alpha(\rho, A)= & -0.8135+0.6189 A+1.188 \rho-0.7935 A \cdot \rho \\
& -0.3195 \rho^{2}+0.1205 A \cdot \rho^{2}+0.02337 \rho^{3}
\end{aligned}
$$

As a result, we obtain the nonlinear mixed damping model of the MMD by substituting all the identified parameters to equation (13):

$$
\begin{aligned}
& F(y(t), \dot{y}(t), t)=\left(3.137 e-5 A^{0.05217} \rho^{9.108}\right) y(t) \\
&+\left(\begin{array}{c}
-836.7-367.4 \cdot A+975.6 \rho \\
-183.1 A^{2}-327.3 A \cdot \rho-314.7 \rho^{2} \\
+2760 A^{3}+1299 A^{2} \cdot \rho \\
-69.43 A \cdot \rho^{2}+36.29 \rho^{3}
\end{array}\right) y^{3}(t) \\
&+\left(\begin{array}{c}
-6.705+9.596 A+6.924 \rho \\
+0.7754 A^{2}-7.209 A \cdot \rho-2.286 \rho^{2} \\
+0.1469 A^{3}+1.297 A^{2} \cdot \rho \\
+0.2459 A \cdot \rho^{2}
\end{array}\right)|\dot{y}(t)|^{-0.3195 \rho^{2}+0.1205 A \cdot \rho^{2}+0.02337 \rho^{3}}-0.8135+0.6189 A+1.188 \rho-0.7935 A \cdot \rho \\
& \operatorname{sgn}(\dot{y}(t)) .
\end{aligned}
$$

4.4. Effect of Mesh Density on the Hysteresis Behavior of $M M D s$. Figure 8 illustrates the effect of mesh density on the hysteresis loop of MMDs. The crisscross angle $\theta$ and wire diameter are $60^{\circ}$ and 0.3 , respectively, while the mesh density is varying from 2.0 to 4.0 . The hysteresis loops exhibit a counterclockwise rotation as the mesh density increases, which indicates that a stiffness change is produced. The area of the hysteresis loop differs from one to another, suggesting that the energy dissipation of MMDs is affected by the mesh density as well.

To quantify the testing data, the stiffness and loss factors are depicted as functions of mesh density in Figure 9. Both 


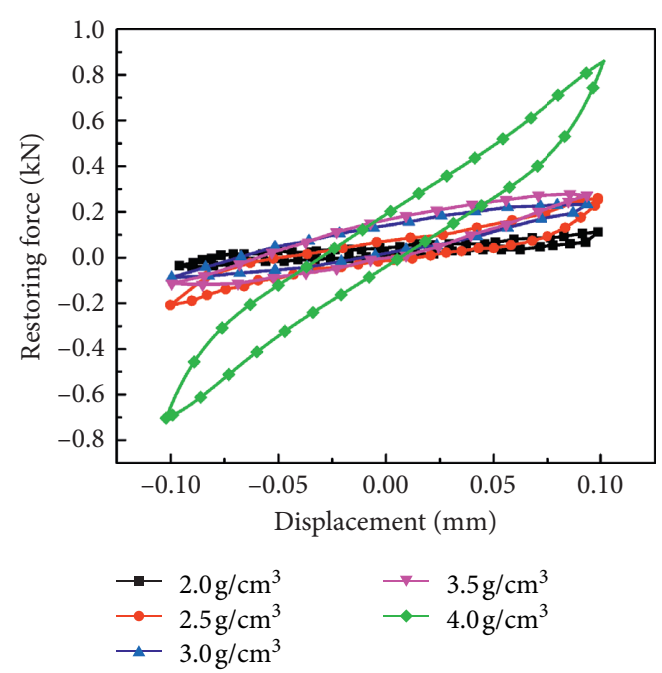

FIGURE 8: Effect of mesh density on the hysteresis behavior of MMDs.

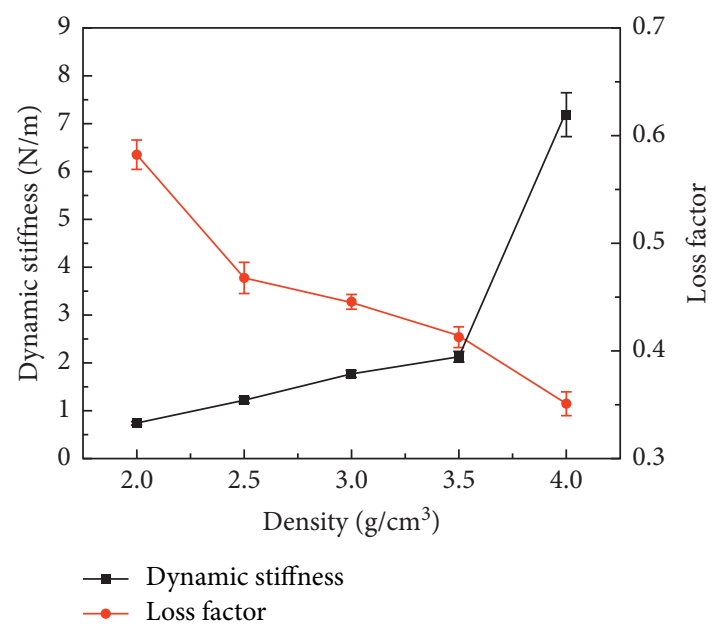

FIgURe 9: Variation of dynamic stiffness and loss factor with mesh density.

stiffness and loss factors vary with mesh density monotonically but demonstrate opposite tendencies. The increasing mesh density results in a rise in stiffness but, on the contrary, a drop in loss factors. The increased mesh density leads to more compacted wires and multiplied dry friction joints in unit volume. On the one hand, this reduces the length and increases the stiffness of the microbeams, the basic components that constitute the entire metal mesh material according to [21]. On the other hand, the motion of one single pair of friction joints is restricted to a smaller range due to the densified helix wires, thereby attenuating the friction dissipation. The elastic potential, meanwhile, becomes greater owing to the increased stiffness, which gives rise to a lower value of loss factor.

4.5. Effect of Crisscross Angle $\theta$ on the Hysteresis Behavior of $M M D$. Figure 10 shows the effect of crisscross angle on the hysteresis loop of MMDs. The mesh density and wire

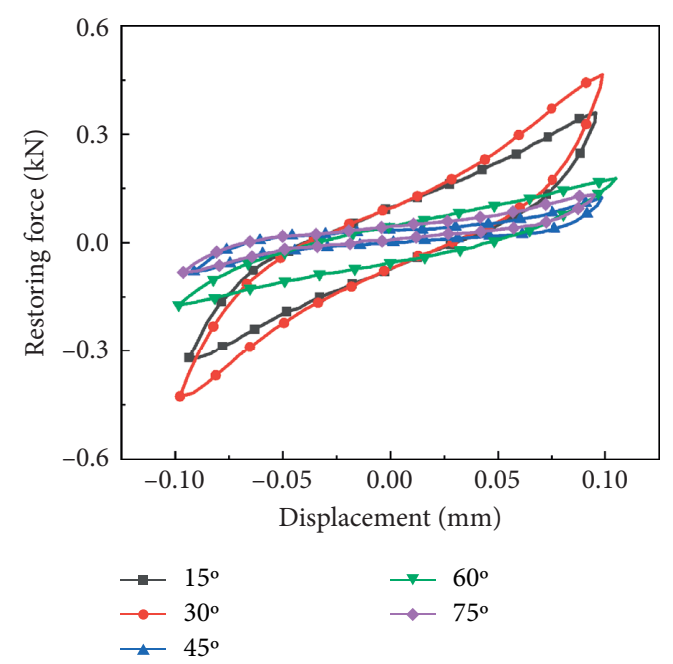

FIGURE 10: Effect of crisscross angle on the hysteresis behaviors of MMDs.

diameter are kept at fixed values of $3.0 \mathrm{~g} / \mathrm{cm}^{3}$ and $0.3 \mathrm{~mm}$, respectively, while the crisscross angle is varying from $15^{\circ}$ to $75^{\circ}$. The hysteresis loops spin clockwise as the crisscross angle increases, indicating a different stiffness change in contrast to Figure 8.

The stiffness and loss factors are shown as functions of the crisscross angle in Figure 11. The stiffness of MMDs decreases rapidly from 3.5 to 1.0 as the angle increases from $15^{\circ}$ to $45^{\circ}$ and then tends to stay at a low level. The crisscross angle only produces a slight change to the loss factor which slowly declines from 0.5 to 0.35 and then bounces back to a local maximum.

4.6. Effect of Wire Diameter on the Hysteresis Behavior of $M M D s$. Figure 12 shows how the hysteresis loops evolve with the wire diameter. The extent to which the peak restoring force varies is much smaller as compared to Figures 8 and 10 , which proves again that the wire diameter plays a relatively minor role in hysteresis behavior. The hysteresis cycles just rotate at a small angle in a counterclockwise direction, which turns out to be a little improvement in stiffness as shown in Figure 13. On the one hand, the enlarged diameter increases the rigidity of every single wire, hence a higher stiffness of the entire metal mesh ring. On the other hand, the number of friction joints is reduced to some extent due to the shortened length of the helix wire, leading to a slight reduction in the loss factor.

\subsection{Comparison of FE Simulation and Mixed Damping Model.}

The hysteresis loops simulated by the FE method and a mixed damping model are compared with experimental results under different conditions. Figure 14 demonstrates the simulated and experimental results of hysteresis loops that evolve with different mesh densities. Apparently, both the FE approach and the mixed damping method can capture the "counterclockwise spin" that is representative of the increasing stiffness of the MMDs. However, the FE model 


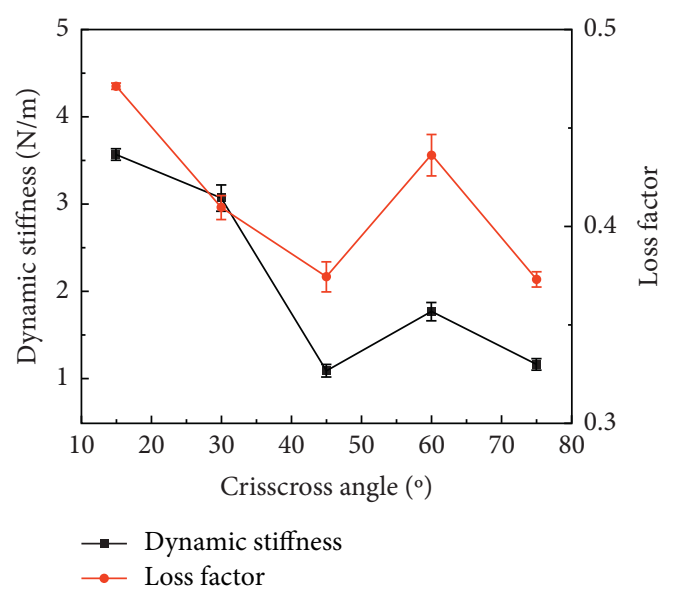

FIGURE 11: Variation of dynamic stiffness and loss factor with the crisscross angle.

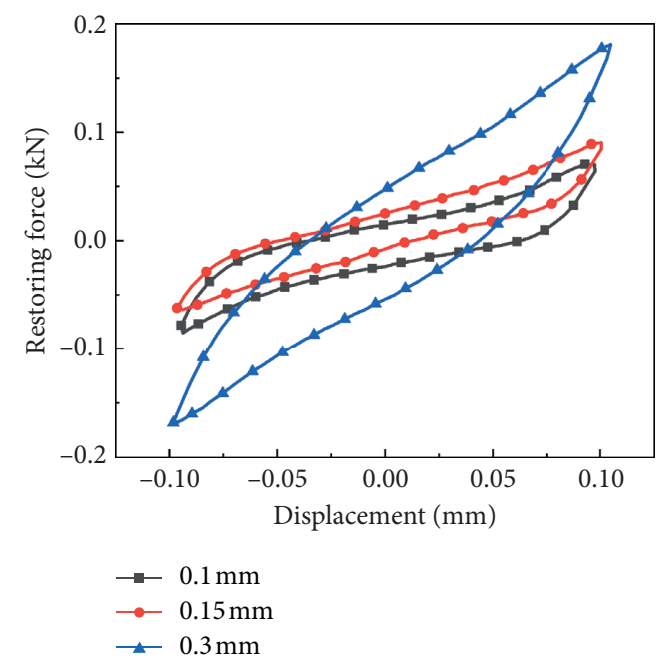

FIGURE 12: Effect of wire diameter on the hysteresis behavior of MMDs.

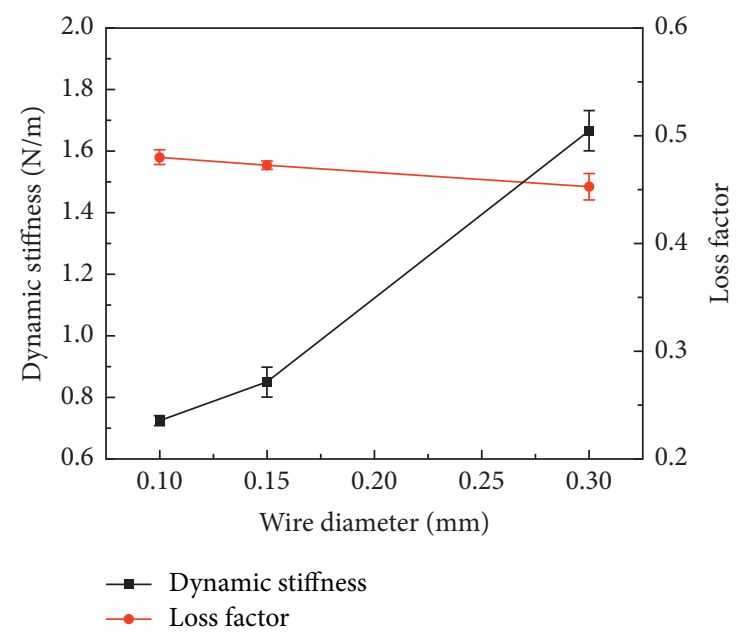

FIgURE 13: Variation of stiffness and loss factor with the wire diameter. achieves an exaggerated area of the hysteresis loop in the case of mesh density $4.0 \mathrm{~g} / \mathrm{cm}^{3}$, which is radically different from the experimental result. In contrast to that, the mixed damping model is well in accordance with the experimental hysteresis loops in every single case of mesh density. Even the inflection points that are omitted in FE results can be correctly predicted by the mixed damping model.

The RMS error between the simulation and experimental hysteresis loops is evaluated using the following equation:

$$
\sigma=\sqrt{\frac{1}{N} \sum_{i=1}^{N}\left(\frac{E_{\text {simul }}(i)-E_{\text {exp }}(i)}{E_{\exp }(i)}\right)^{2}}
$$

and then plotted as histogram shown in Figure 15. For all the cases of mesh densities, the error of the mixed damping model is steadily kept at a level lower than 0.375 whereas the FE approach presents a higher error 2 30 times larger than the mixed damping model.

Figure 16 demonstrates how the simulated stiffness and loss factor respond to the variation of mesh density. The mixed damping model predicts the way experimental stiffness and loss factor change with mesh density. However, the FE results deviate significantly from the experimental results, especially the simulated values of the loss factor.

Apart from mesh density, displacement amplitude is another key factor that affects the hysteresis behavior. Figure 17 compares the simulated and experimental results of hysteresis loops with different loading amplitudes. It can be found that the inflection points are still neglected in the FE results which crudely depict the hysteresis loop as an ellipse. Furthermore, the two modeling approaches present nearly opposite ways in the prediction of stiffness changes, as shown in Figure 18(a). The stiffness values simulated by the mixed damping model, which show good agreement with the experiment, decrease with loading amplitude rapidly first and then slowly approach a steady value. On the contrary, the stiffness values simulated by the FE model demonstrate a concave feature. That is, the decreasing tendency of calculated stiffness becomes even larger as the loading amplitude increases. The FE model also fails to predict the way loss factors vary with the amplitude. As shown in Figure 18(b), a considerable difference can be observed between the FE results and the experimental values of the loss factor.

\section{Discussion}

5.1. Comparing the Implementation of the Two Different Modeling Methods. Two different modeling approaches are adopted in the present work, aiming to predict how the dynamic behavior of MMDs responds to excitation conditions and fabrication parameters. The first approach is FE modeling based on the transient structural module of the ANSYS workbench. External excitation conditions and material parameters are required for the input, and the material model plays a critical role to solve the dynamic response of the MMDs. On the contrary, the material model is no longer required for the mixed damping approach, the second method adopted in the present work. 

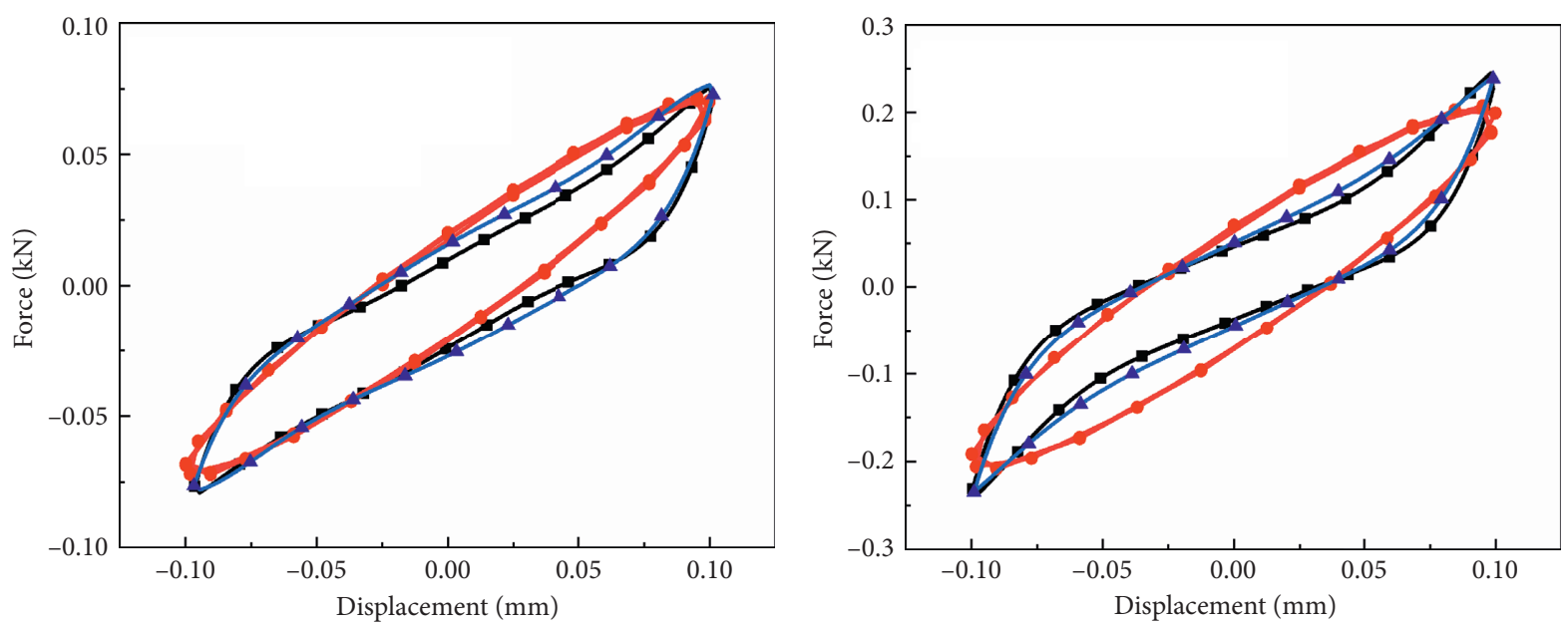

$\rightarrow$ Experiment

- FE model

— Mixed damping model

$\rightarrow-$ Experiment

$\rightarrow$ FE model

^ Mixed damping model

(a)
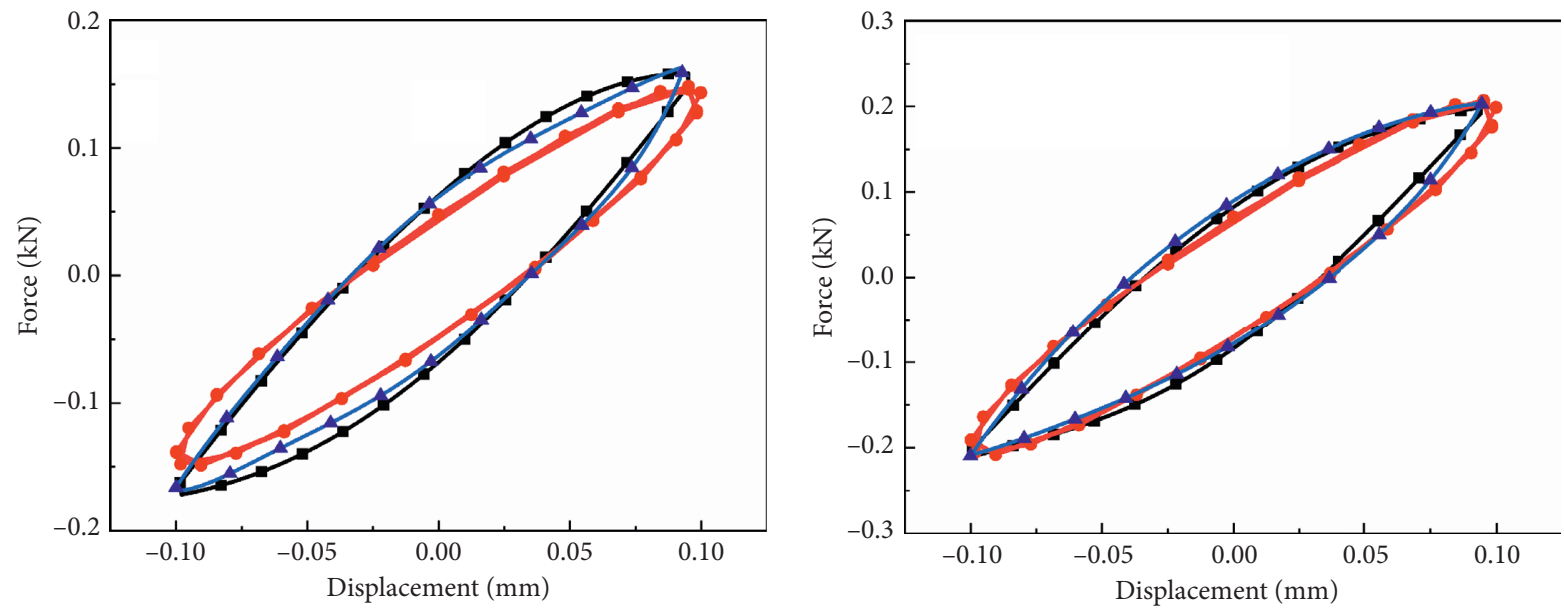

$\rightarrow-$ Experiment

$\rightarrow$ Experiment

$\longrightarrow$ FE model

$\neg$ Mixed damping model

- FE model

$\_$Mixed damping model

(c)

(d)

FIGURE 14: Continued. 


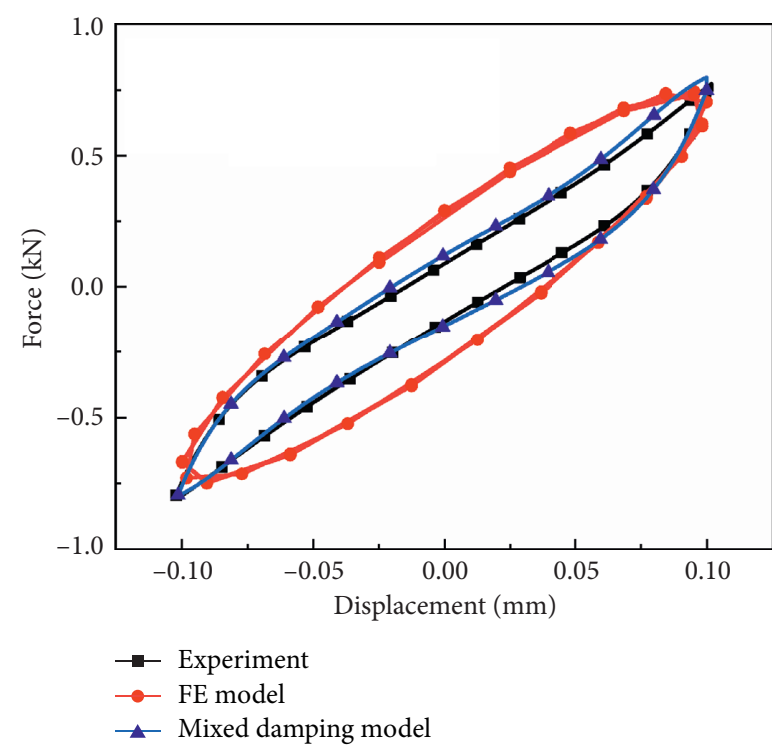

(e)

Figure 14: Simulated and experimental hysteresis loops of MMDs with different densities. (a) Mesh density $2.0 \mathrm{~g} / \mathrm{cm}^{3}$. (b) Mesh density $2.5 \mathrm{~g} / \mathrm{cm}^{3}$. (c) Mesh density $3.0 \mathrm{~g} / \mathrm{cm}^{3}$. (d) Mesh density $3.5 \mathrm{~g} / \mathrm{cm}^{3}$. (e) Mesh density $4.0 \mathrm{~g} / \mathrm{cm}^{3}$.

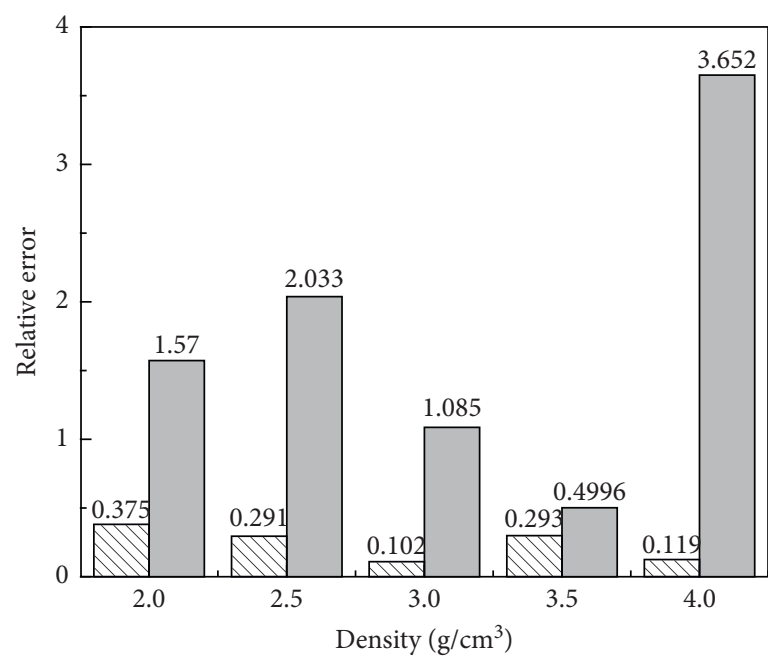

MIV Mixed damping model

FE model

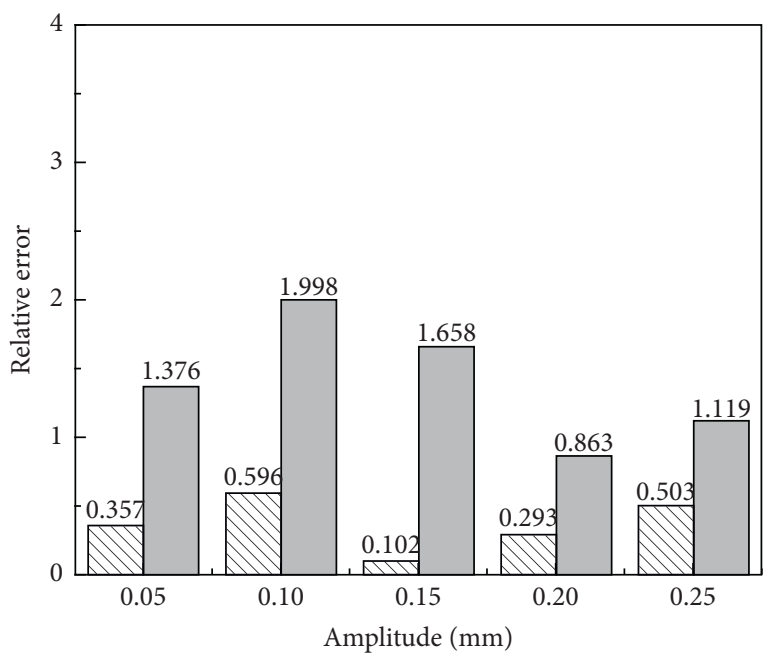

Mixed damping model

FE model

(a)

(b)

FIGURE 15: RMS error between simulated and experimental results of hysteresis loops. (a) Error varying with mesh density. (b) Error varying with amplitude.

Phenomenological parameters describing mechanical behaviors including nonlinear restoring and damping are simply identified by fitting hysteresis loops against mathematical models.

It is noteworthy that different sets of material parameters are required for the FE model when MMDs are fabricated with various mesh densities, as is reflected in Table 4. In fact, metal mesh material which consists of a great number of helix microstructures is a kind of structure instead of material. Once the mesh density is changed, the microstructures within the MMDs are modified accordingly. As a result, a unified set of material parameters for metal mesh materials are hard to be obtained, and the FE model calibrated for one density would fail to capture the dynamic mechanical behavior when the density is changed to another. In addition to that, the viscoelastic material model is unable to simulate critical features that are revealed in the experimental results. For 


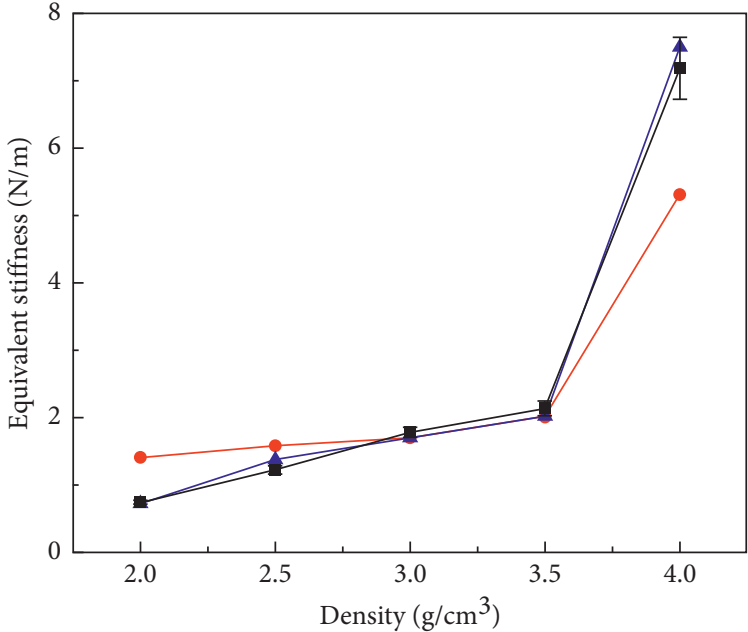

- Experiment

$\longrightarrow$ FE model

$\leftarrow$ Mixed damping model

(a)

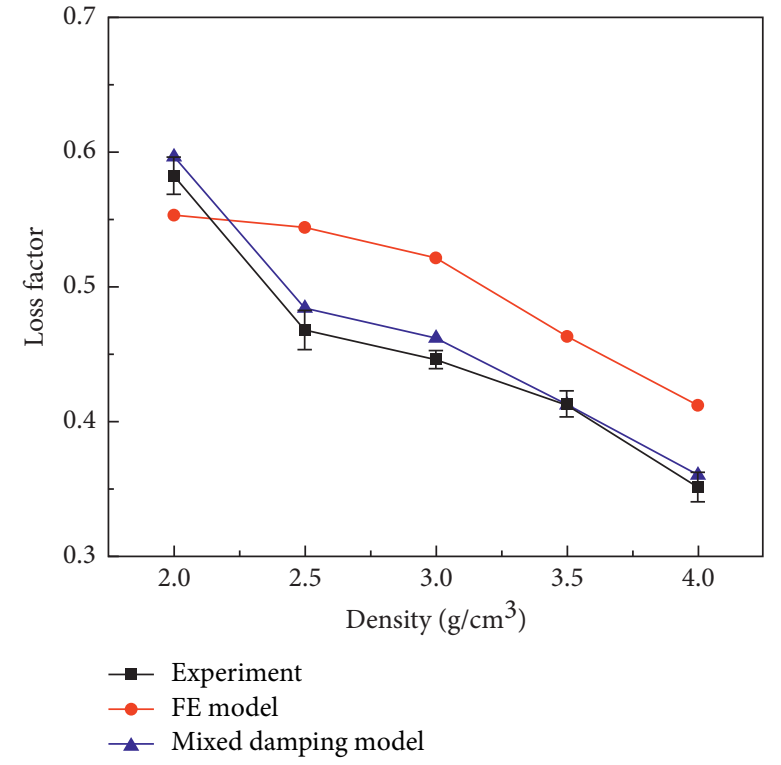

(b)

FIGURE 16: Simulated values of (a) stiffness and (b) loss factor plotted as a function of mesh density.
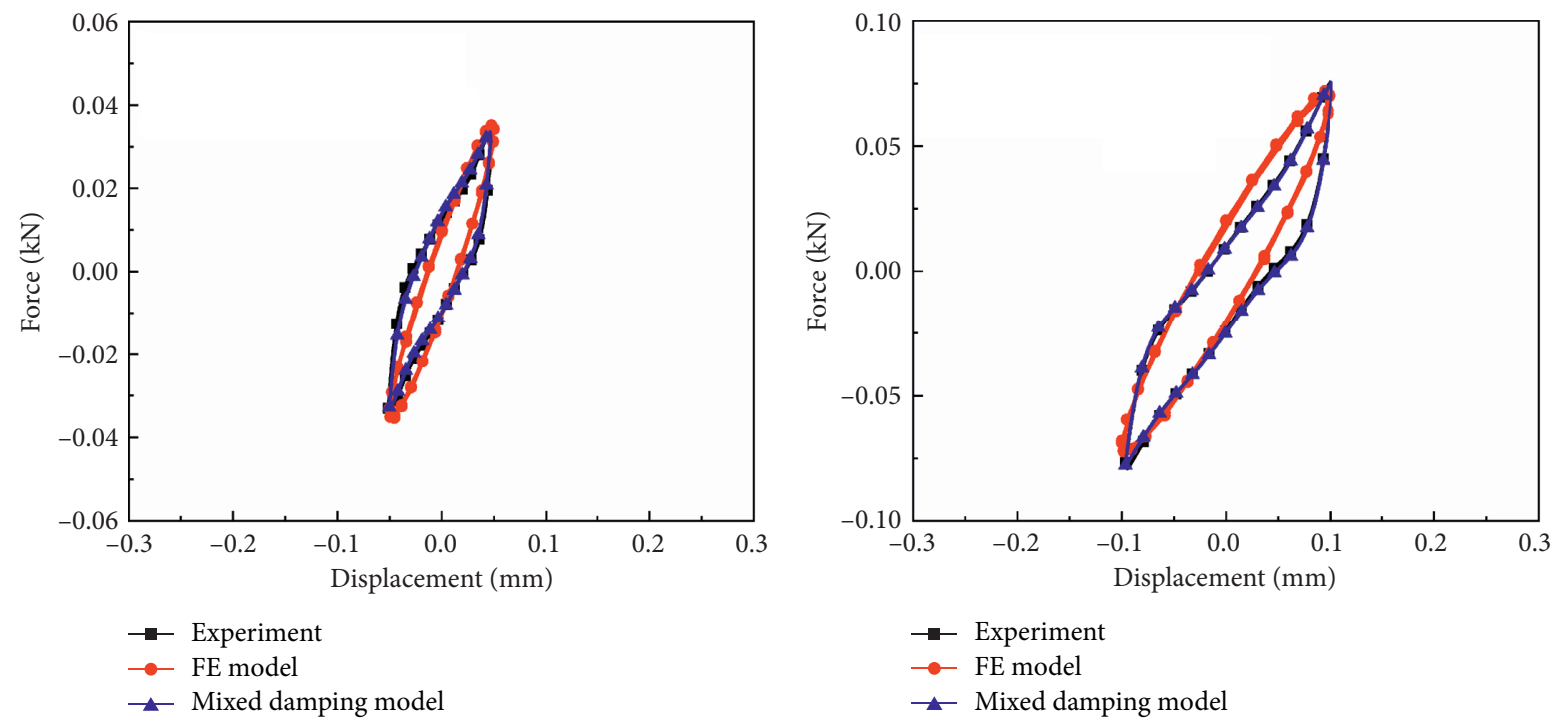

$\rightarrow$ Experiment

$\neg$ Mixed damping model

(b)

(a)

Figure 17: Continued. 


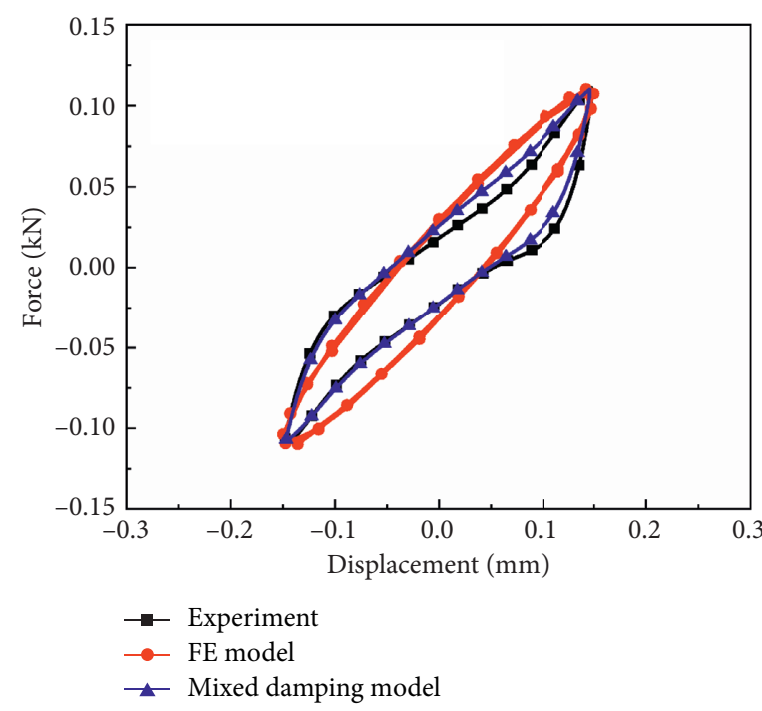

(c)

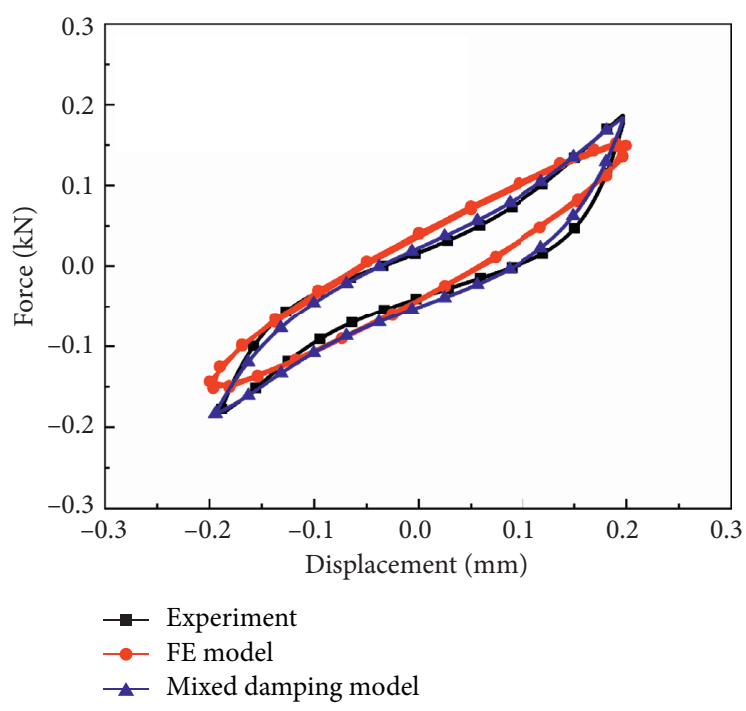

(d)

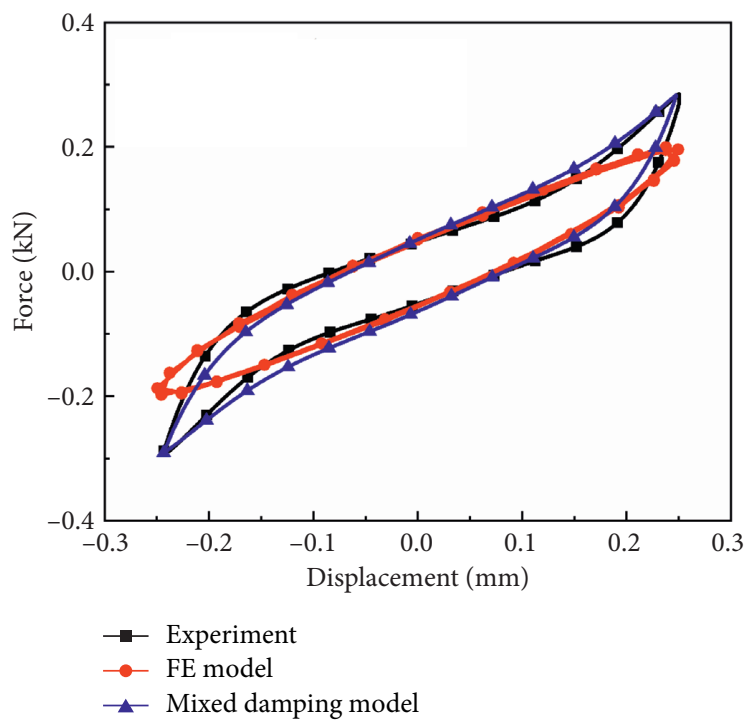

(e)

FIgURE 17: Simulated and experimental hysteresis loops of MMDs of mesh density $2.0 \mathrm{~g} / \mathrm{cm}^{3}$ excited with different amplitudes. (a) Amplitude $0.05 \mathrm{~mm}$. (b) Amplitude $0.1 \mathrm{~mm}$. (c) Amplitude $0.15 \mathrm{~mm}$. (d) Amplitude $0.2 \mathrm{~mm}$. (e) Amplitude $0.25 \mathrm{~mm}$.

instance, the inflection points are missing from the hysteresis loops. In contrast to the FE model, the mixed damping approach can capture the details by taking advantage of identifying the first-order and the thirdorder stiffness coefficient, although those are not parameters correlated with any physical properties of the material.

The damping mixed approach proves a better solution to describe the dynamic behaviors as compared to the $\mathrm{FE}$ model. Although a heavy reliance on experimental results is inevitable, this approach does reflect the internal essence of the experimental phenomenon. The first-order and thirdorder stiffness coefficients are heavily dependent on mesh density other than motion amplitude. This proves again the importance of mesh density to the hysteresis behaviors of the metal mesh material.
In future work, a user-defined material subroutine will be developed to define how the microstructures inside the MMDs change with the mesh density and to modify the constitutive law by relating the microstructures to the viscoelasticity. In so doing, a predictive FE model for MMDs can be implemented without heavy reliance on experimental results.

\subsection{Applicability of the Methodology of the Present Work.} A set of parameters representative of the typical fabrication procedure of ring-shaped MMD is selected in this paper to conduct all the tests and simulations in consideration of the fabrication quality of the ring-shaped MMD. For instance, a crisscross angle smaller than $10^{\circ}$ will weaken the interlocked structures between the different layers of the wrapped helix wire, which results in structural failure under the vibration 


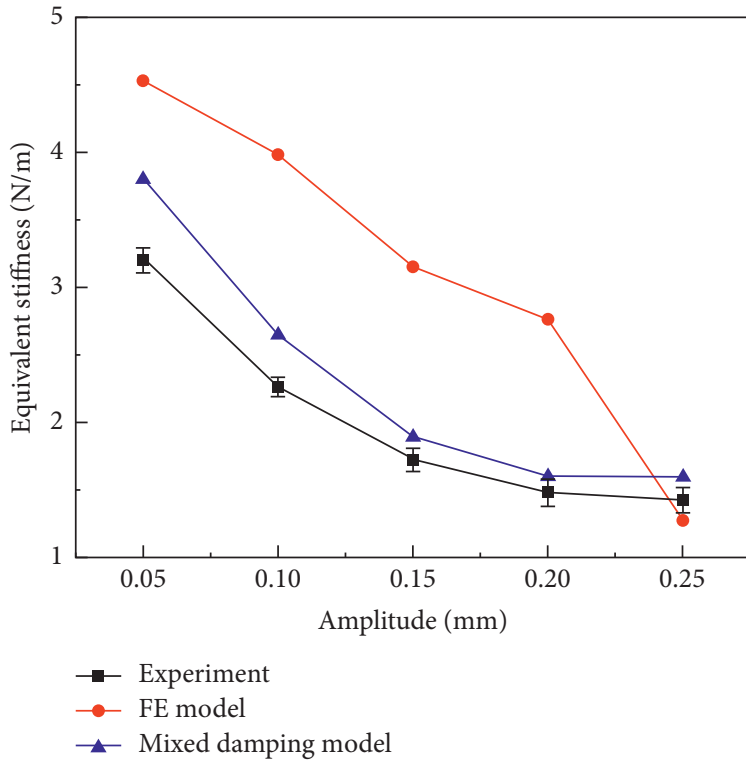

(a)

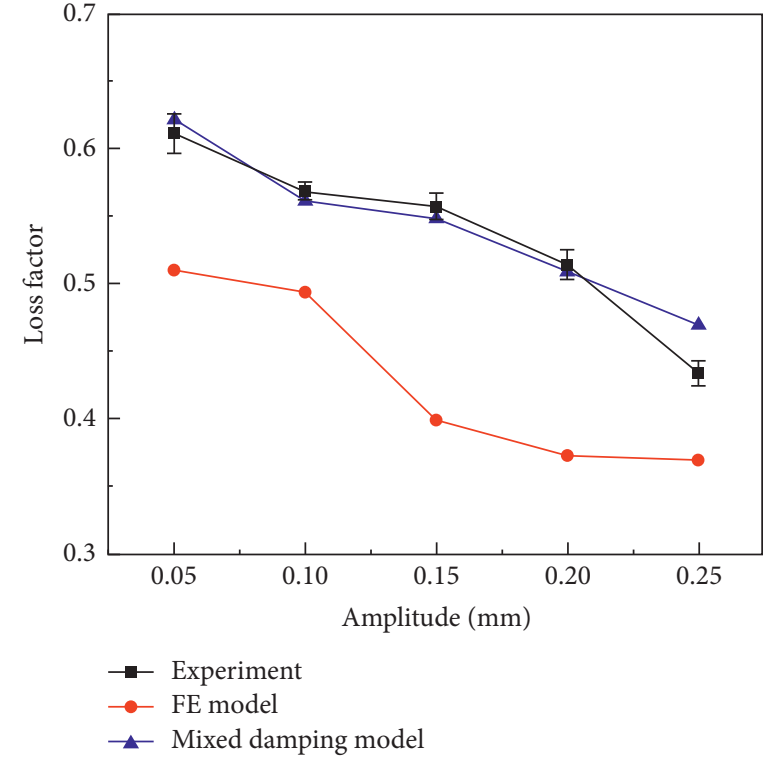

(b)

Figure 18: Simulated values of (a) stiffness and (b) loss factor plotted as a function of amplitude.

environment. In addition, an extreme wire density smaller than $1.0 \mathrm{~g} / \mathrm{cm}^{3}$ or larger than $5.0 \mathrm{~g} / \mathrm{cm}^{3}$ would lead to either poor loading capacity of the MMD or higher fabrication cost.

Although a limited range of fabrication parameters is employed in this paper, one can still extend the application of the mixed damping method to situations outside the given range of the parameters in Table 1 . As a modeling technique based on system identification theory, a mathematic relation between the external excitations and the response of the MMD can be constructed by decomposing the nonlinear restoring force in terms of elastic force and damping force and then identifying the free parameters involved in the "grey box model." Therefore, the methodology is applicable as long as the force-displacement inputs and outputs are given. As reported in [31], a crisscross angle as high as $90^{\circ}$ is used by the authors to improve the durability of the ringshaped MMDs under fatigue loading cycles. Metal rubber sheets as thin as $2.5 \mathrm{~mm}$ were fabricated with a density of $1.8 \mathrm{~g} / \mathrm{cm}^{3}$ and wrapped around the corrugated pipe to isolate vibrations [30]. In both works where the fabrication parameters of MMDs do not fall within the given range of Table 1, the mixed damping method was successfully applied to predict the dynamic mechanical behavior of the metal rubber devices. Apart from that, the mathematical function of dynamic response versus excitation conditions or even environmental conditions can be conveniently established via the mixed damping method. For instance, the dynamic response of MMD was built as a function of restoring force versus excitation frequency and ambient temperature in the published work [25].

\section{Conclusions}

In the present paper, efforts are directed toward modeling the dynamic behavior of metal mesh damper subjected to sinusoidal excitation. Dynamic mechanical tests are conducted as well to verify the predicted results. Conclusions can be drawn as follows:

(1) By using orthogonal test design and ranging analysis, the effect of fabrication procedure on the dynamic behavior of the MMD is evaluated by ordering the significance of every single fabrication parameter, i.e., mesh density $>$ crisscross angle $>$ wire diameter. The single-factor test indicates that the maximum variation of the dynamic stiffness and loss factor caused by adjusting the mesh density is 6.9 and 6.2 times higher than that caused by changing the wire diameter

(2) As revealed in the experiments, the three fabrication parameters influence the performance of MMDs in different ways. Increasing the mesh density leads to a higher dynamic stiffness but a lower loss factor. A greater crisscross angle results in a decrease in dynamic stiffness and loss factor. Using thicker wire to fabricate the MMD only increases its dynamic stiffness and has little effect on the loss factor

(3) The predictive accuracy of the FE method and the mixed damping approach is assessed by comparing the calculated results of hysteresis loops against experimental ones. The mixed damping approach turns out to exhibit higher agreement with the experimental results. The shape of the hysteresis loop can be depicted more accurately by the mixed damping approach by taking advantage of identifying key parameters including the stiffness coefficient, the damping coefficient, and the damping component factor. Furthermore, this approach can automatically respond to the change of fabrication parameters and external excitation conditions by 
defining internal variables as functions of the mesh density and the motion amplitude

(4) Although optimization of fabrication parameters is not concerned in this paper, the mixed damping model proposed in the present work can help construct the desired MMDs when design requirements are given. Since the model automatically responds to the change of mesh density and excitation amplitude, one can use it reversely to determine which mesh density should be chosen when specific loss factor and dynamic stiffness of the MMD are required

\section{Data Availability}

The data used to support the findings of this study are available from the corresponding author upon request.

\section{Conflicts of Interest}

The authors declare that they have no conflicts of interest.

\section{Acknowledgments}

This work was supported by the Natural Science Foundation of Fujian Province, China (Grant no. 2018J01763), Natural Science Foundation of China (Grant no. 12002088), Education and Scientific Research Foundation for Young Teachers in Fujian Province (Grant no. JAT190008), and Starting Grants of Fuzhou University (Grant no. GXRC20017).

\section{References}

[1] H.-K. Jeong, J.-H. Han, S.-H. Youn, and J. Lee, "Frequency tunable vibration and shock isolator using shape memory alloy wire actuator," Journal of Intelligent Material Systems and Structures, vol. 25, no. 7, pp. 908-919, 2013.

[2] K. Komatsu and H. Uchida, "Microvibration in spacecraft," Mechanical Engineering Reviews, vol. 1, no. 2, Article ID SE0010, 2014.

[3] X. Cao, C. Wei, J. Liang, and L. Wang, "Design and dynamic analysis of metal rubber isolators between satellite and carrier rocket system," Mechanical Sciences, vol. 10, no. 1, pp. 71-78, 2019.

[4] H. Fu, Z. Hua, L. Zou, Y. Wang, and J. Ye, "Combined stiffness characteristic of metal rubber material under vibration loads," Proceedings of the Institution of Mechanical Engineers-Part C: Journal of Mechanical Engineering Science, vol. 233, no. 17, pp. 6076-6088, 2019.

[5] S.-C. Kwon, S.-H. Jeon, and H.-U. Oh, "Performance evaluation of spaceborne cryocooler micro-vibration isolation system employing pseudoelastic SMA mesh washer," Cryogenics, vol. 67, pp. 19-27, 2015.

[6] S. C. Kwon, S. H. Jeon, and H. U. Oh, "Performance investigation of a novel pseudoelastic SMA mesh washer gear wheel with micro-jitter attenuation capability," Smart Materials and Structures, vol. 25, no. 5, Article ID 055004, 2016.

[7] H. U. Oh, S. H. Jeon, T. H. Kim et al., "Experimental feasibility study for micro-jitter attenuation of stepper-actuated X-band antenna-pointing mechanism by using pseudoelastic SMA mesh washer," Smart Materials and Structures, vol. 24, no. 4, Article ID 045010, 2015.
[8] H. U. Oh, S. C. Kwon, and S. H. Youn, "Characteristics of spaceborne cooler passive vibration isolator by using a compressed shape memory alloy mesh washer," Smart Materials and Structures, vol. 24, no. 1, Article ID 015009, 2014.

[9] S. H. Youn, Y. S. Jang, and J. H. Han, "Development of a threeaxis hybrid mesh isolator using the pseudoelasticity of a shape memory alloy," Smart Materials and Structures, vol. 20, no. 7, Article ID 075017, 2011.

[10] S.-H. Youn, Y.-S. Jang, and J.-H. Han, "Compressed mesh washer isolators using the pseudoelasticity of SMA for pyroshock attenuation," Journal of Intelligent Material Systems and Structures, vol. 21, no. 4, pp. 407-421, 2009.

[11] J. M. Vance, Rotordynamics of Turbomachinery, John Wiley \& Sons, New York, NY, USA, 1987.

[12] B. H. Ertas, "Compliant hybrid journal bearings using integral wire mesh dampers," Journal of Engineering for Gas Turbines \& Power, vol. 131, no. 1-11, Article ID 022503, 2009.

[13] B. Ertas, H. Luo, and D. Hallman, "Dynamic characteristics of shape memory alloy metal mesh dampers," in Procedeedings of the 50th AIAA/ASME/ASCE/AHS/ASC Structures, Structural Dynamics, and Materials Conference, p. 2521, Palm Springs, CA, USA, May 2009.

[14] B. H. Ertas and H. Luo, "Nonlinear dynamic characterization of oil-free wire mesh dampers," Journal of Engineering for Gas Turbines \& Power, vol. 130, no. 3, p. 032503, 2008.

[15] A. M. Zhizhkin, V. A. Zrelov, V. V. Zrelov, A. Y. Ardakov, and A. A. Osipov, "Rotor s based on a metal-rubber elastic porous material for turbomachinery," Journal of Friction and Wear, vol. 39, no. 3, pp. 259-263, 2018.

[16] Y. Ma, Q. Zhang, D. Zhang, F. Scarpa, B. Liu, and J. Hong, "Tuning the vibration of a rotor with shape memory alloy metal rubber supports," Journal of Sound and Vibration, vol. 351, pp. 1-16, 2015.

[17] L. San Andrés, T. A. Chirathadam, and T.-H. Kim, "Measurement of structural stiffness and damping coefficients in a metal mesh foil bearing," Journal of Engineering for Gas Turbines \& Power, vol. 132, no. 3, 2009.

[18] Y. B. Lee, C. H. Kim, T. H. Kim et al., "Effects of mesh density on static load performance of metal mesh gas foil bearings," Journal of Engineering for Gas Turbines \& Power, vol. 134, no. 1, 2011.

[19] K. Ryu and H. Yi, "Wire mesh dampers for semi-floating ring bearings in automotive turbochargers: measurements of structural stiffness and damping parameters," Energies, vol. 11, no. 4, p. 812, 2018.

[20] H. Ao, Y. Ma, X. Wang, and H. Jiang, "Modelling and prediction of lifetime of metal rubber isolator under random vibration," in Mechanisms and Machine Science, pp. 376-384, Springer, Berlin, Germany, 2019.

[21] F. L. Cao, H. B. Bai, D. W. Li et al., "A constitutive model of metal rubber for hysteresis characteristics based on a mesomechanical method," Rare Metal Materials and Engineering, vol. 45, no. 1, pp. 1-6, 2016.

[22] V. V. Choudhry and J. M. Vance, "Design equations for wire mesh bearing dampers in turbomachinery," in Power for Land, Sea, and Air, pp. 807-814, ASME Turbo Expo, New York, NY, USA, 2005.

[23] E. M. Al-Khateeb, Design, Modeling and Experimental Investigation of Wire Mesh Vibration Dampers, Texas A \& M University, Station, TX, USA, 2002.

[24] J. F. Hou, H. B. Bai, and D. W. Li, "Damping capacity measurement of elastic porous wire-mesh material in wide temperature range," Journal of Materials Processing Technology, vol. 206, no. 1, pp. 412-418, 2008. 
[25] F. Jiang, Z. Ding, Y. Wu, H. Bai, Y. Shao, and B. Zi, "Energy dissipation characteristics and parameter identification of symmetrically coated damping structure of pipelines under different temperature environment," Symmetry, vol. 12, no. 8, p. $1283,2020$.

[26] A. Pérez, D. Ferreño, I. A. Carrascal et al., "Metal cushion dampers for railway applications: a review," Construction and Building Materials, vol. 238, Article ID 117711, 2020.

[27] Z. Y. Ren, Q. Chen, H. Bai et al., "Study on damping energy dissipation characteristics of cylindrical metal rubber in nonforming direction," Advances in Materials Science and Engineering, vol. 2018, Article ID 5014789, 10 pages, 2018.

[28] H. Z. Cheng, Research on of Static Characteristics and Vibration Isolation Mechanism of Metal Rubber Material, Harbin Engineering University, Harbin, China, 2015.

[29] J. Wu, C. Liu, H. Jiang, and Z Wang, "Numerical modeling and experimental testing analysis of assembled rubber metal isolator," Science Progress, vol. 103, no. 3, Article ID 36850420956985, 2020.

[30] K. N. Wu, H. B. Bai, X. Xue et al., "Energy dissipation characteristics and dynamic modeling of the coated damping structure for metal rubber of bellows," Metals, vol. 8, no. 7, Article ID 562, 2018.

[31] P. Yang, H. Bai, X. Xue, K. Xiao, and X. Zhao, "Vibration reliability characterization and damping capability of annular periodic metal rubber in the non-molding direction," $M e$ chanical Systems and Signal Processing, vol. 132, pp. 622-639, 2019. 